\title{
Varietäten und Sprachkontakt in rätoromanischen SMS*
}

\author{
Matthias Grünert (Zürich)
}

\begin{abstract}
As a minority language in Switzerland, the Romansh of the Grisons is exposed to pressure from the dominant language in its region, namely (Swiss) German. Consequently, it has not developed a generally accepted standardized written form, being above all a spoken language, which cedes many written functions to the dominant language. These premises entail a complex spectrum of language variation in informal written use of Romansh in SMScommunication. This variation includes deviations from regional written norms, traits of (dialectal) orality, interference of (Swiss) German, and code-switching to (Swiss) German. Language contact is evident in the fact that Romansh is used for part of an SMS whereas another part of the same SMS sent by the same person may be written in one or even in two varieties of the majority language, i.e. German and Swiss German. Some speakers of Romansh, mainly people who live outside of the traditional linguistic territory, do not write in Romansh at all. It should be noted that the users of Romansh in SMS-communication who participated in this research project are mainly women with university-level educations who are working in the tertiary sector.
\end{abstract}

\section{Das erste SMS-Korpus zum Rätoromanischen Graubündens}

Im Rahmen des Projektes sms4science ist das erste Korpus von SMS, die im Rätoromanischen Graubündens ${ }^{1}$ verfasst sind, zustande gekommen. Dieses Korpus erlaubt erstmals grundsätzliche Beobachtungen zur SMS-Kommunikation in der vierten Landessprache der Schweiz, die bisher nie Gegenstand wissenschaftlicher Untersuchungen zur computervermittelten Kommunikation war, auch wenn - insbesondere zur Kommunikation im Internet - reichlich interessante Daten vorhanden wären. ${ }^{2}$

\footnotetext{
* Christa Dürscheid (Zürich) danke ich für die Überprüfung meines Textes und ihre wertvollen Anregungen. Zu Dank bin ich auch folgenden Personen, für ihre Hinweise zum Text und ihre sprachlichen Auskünfte, verpflichtet: Sidonia Klainguti (Samedan), Silvana Derungs (Chur/Surcasti), Clau Solèr (Chur/Lumbrein), Clà Riatsch (Bern/Ramosch), Ricarda Liver (Lützelflüh), Barbara Strebel (Hombrechtikon/Chur) und Valeria Badilatti (Zürich/Zuoz).

${ }^{1}$ Dem Terminus "Bündnerromanisch", der in der Wissenschaft als Bezeichnung für das Rätoromanische Graubündens verwendet wird, ziehen wir für unsere Ausführungen den in der Deutschschweiz üblichen Terminus "Rätoromanisch" (ohne weitere Bestimmung) vor. Mit diesem Terminus wurden auch die rätoromanischsprachigen Teilnehmenden unserer Erhebung in dem auf Deutsch verfassten Fragebogen konfrontiert, und sie verwendeten selbst diesen Terminus in ihren Bemerkungen. In der Wissenschaft wird "Rätoromanisch" zur Bezeichnung eines Typs romanischer Sprachen gebraucht, der neben dem Bündnerromanischen das in den italienischen Provinzen Bozen, Trient und Belluno gesprochene Dolomitenladinische sowie das im Nordosten Italiens gesprochene Friulanische einschliesst (cf. Liver 2010: 15). Die Sprachbezeichnung "Bündnerromanisch" wird jedoch im Alltag nie verwendet (sie verweist auch auf eine Sprachklassifikation, die für die Sprachbenutzerinnen und -benutzer nicht von Belang ist). Im Kanton Graubünden - nicht nur in der Alltagskommunikation, sondern auch in den Medien und in einem Teil der amtlichen Dokumente - lautet das Glottonym sehr unspezifisch schlichtweg "Romanisch".

${ }^{2}$ Cf. dazu einige Hinweise am Schluss dieses Beitrags.
} 
Mit seinen bisher identifizierten 214 in Rätoromanisch verfassten SMS verdient das umfangmässig bescheidene Subkorpus als erste derartige Sammlung besondere Aufmerksamkeit. Was den Umfang des Subkorpus betrifft, ist bemerkenswert, dass der Anteil rätoromanischer SMS am gesamten Schweizer SMS-Korpus dem Anteil der Rätoromanisch Sprechenden an der Schweizer Wohnbevölkerung zumindest annähernd zu entsprechen scheint: 214 von 23'987 SMS, also $0,89 \%$, wurden als rätoromanisch identifiziert; anlässlich der letzten Volkszählung im Jahre 2000 gaben 0,83\% (d.h. 60'561 von den 7'288'010 Personen, die damals die Fragen zu den Sprachen beantworteten) das Rätoromanische als Hauptsprache und/oder als Umgangssprache an (Furer 2005: 30). ${ }^{3}$ Es kann aber nicht ausgeschlossen werden, dass im Laufe der Bearbeitung des Schweizer SMS-Korpus weitere rätoromanische SMS gefunden werden. Die automatische Spracherkennung hat nämlich in einem ersten Schritt nur 113 SMS zu Tage gefördert. Aufgrund der Identifikationsnummern der Verfasserinnen und Verfasser dieser 113 SMS, aufgrund der Angabe des Rätoromanischen als Muttersprache oder als einer im Alltag gesprochenen Sprache (im Fragebogen) sowie mit der Suche häufiger rätoromanischer Wörter im Textkorpus konnten bisher weitere 101 rätoromanische SMS und acht (schweizer)deutsche SMS mit CodeSwitching ins Rätoromanische identifiziert werden.

In unserem Beitrag geben wir zunächst einen Überblick über die sprachzugehörigkeitsbezogene und soziodemographische Zusammensetzung der Gruppe der rätoromanisch Schreibenden und/oder Sprechenden und skizzieren dann einige markante Merkmale des Gebrauchs von - auf verschiedenen Achsen zu situierenden - rätoromanischen Varietäten, wobei den Sprachkontaktphänomenen besondere Aufmerksamkeit zukommen soll. Abschliessend weisen wir auf Perspektiven zur künftigen Forschung hin.

\section{Sprachzugehörigkeit und soziodemographische Daten}

Die insgesamt 214 rätoromanischen SMS wurden von 30 Personen verfasst, von denen 24 den Fragebogen ausgefüllt haben (cf. Tabelle 1 auf der nächsten Seite). 23 dieser 24 Personen haben das Rätoromanische als Muttersprache angegeben (17 ausschliesslich und 6 neben einer anderen Sprache), eine rätoromanisch schreibende Person hat dagegen in der Rubrik zur Muttersprache nur das Schweizerdeutsche genannt.

\footnotetext{
3 Es handelt sich um diejenigen Personen, die mindestens bei einer der drei Fragen zu den Sprachen im Volkszählungsfragebogen (BFS 2000: 2) das Rätoromanische angegeben haben:

- $\quad$ "Welches ist die Sprache, in der Sie denken und die Sie am besten beherrschen?" (8a)

- $\quad$ "Welche Sprache(n) sprechen Sie regelmässig in der Schule, im Erwerbsleben, im Beruf?" (8b $\left.{ }^{1}\right)$

- $\quad$ "Welche Sprache(n) sprechen Sie regelmässig zu Hause, mit den Angehörigen?" $\left(8 b^{2}\right)$

Die Aussagekraft dieser Daten relativiert Furer (1996: 19): "[Ces données] ne concernent que deux domaines, dont un seul, la famille, peut dans un sens ou dans l'autre toucher virtuellement l'ensemble de la population. On peut donc pour chaque langue imaginer nombre de personnes parlant cette langue, même quotidiennement, mais ailleurs que dans les domaines relevés."
} 

$\mathrm{R}=$ Rätoromanisch
Std $=$ Südtirolerdeutsch
$\mathrm{E}=$ Englisch
$\mathrm{D}=$ Deutsch
I $=$ Italienisch
$\mathrm{S}=$ Spanisch
$\mathrm{Sd}=$ Schweizerdeutsch
$\mathrm{F} \quad=$ Französisch
$\mathrm{P}=$ Portugiesisch

\begin{tabular}{|c|c|c|c|c|c|c|c|c|c|c|}
\hline \multicolumn{7}{|c|}{ Verfasserinnen und Verfasser rätoromanischer SMS (nach Alter) } & \multicolumn{4}{|c|}{ Anzahl SMS } \\
\hline 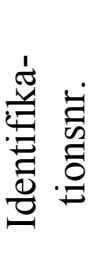 & 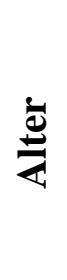 & $\begin{array}{l}\vec{Z} \\
\frac{0}{0} \\
\frac{0}{0} \\
0 \\
0 \\
0 \\
0\end{array}$ & 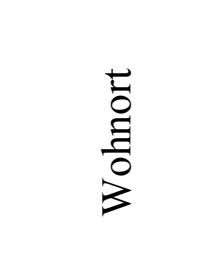 & 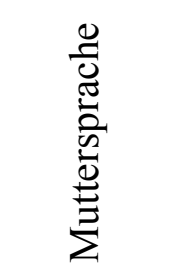 & 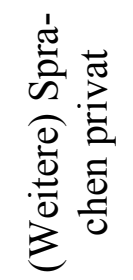 & 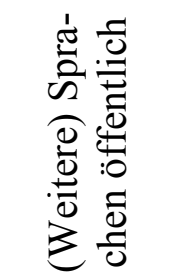 & 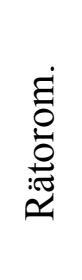 & 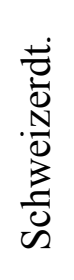 & 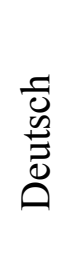 & $\begin{array}{l}\frac{0}{0} \\
\frac{0}{0} \\
\text { त् }\end{array}$ \\
\hline 1198 & 17 & $\mathrm{~W}$ & Pontresina & $\mathrm{R}$ & & & 6 & & & \\
\hline 764 & 18 & $\mathrm{~W}$ & Silvaplana & $\mathrm{R}, \mathrm{Sd}$ & $\mathrm{I}$ & $\mathrm{E}, \mathrm{S}$ & 5 & & & \\
\hline 667 & 19 & $\mathrm{~W}$ & Fex & $\mathrm{R}$ & $\mathrm{Sd}, \mathrm{I}$ & $\mathrm{P}$ & 1 & & & \\
\hline 690 & 20 & $\mathrm{~W}$ & Fex & $\mathrm{Sd}$ & $\mathrm{R}, \mathrm{I}$ & $\mathrm{D}$ & 9 & 44 & & \\
\hline 695 & 20 & $\mathrm{M}$ & Chur & $\mathrm{R}$ & & $\begin{array}{c}\mathrm{Sd}, \mathrm{D}, \mathrm{R}, \\
\mathrm{E}\end{array}$ & 1 & 12 & & $2 \mathrm{E}$ \\
\hline 687 & 21 & $\mathrm{~W}$ & Zug & $\mathrm{R}$ & & $\mathrm{Sd}$ & 16 & & & \\
\hline 733 & 21 & $\mathrm{~W}$ & Curaglia & $\mathrm{R}$ & $\mathrm{R}$ & $\mathrm{R}, \mathrm{D}, \mathrm{I}$ & 6 & & & \\
\hline 723 & 22 & $\mathrm{~W}$ & Zuoz & $\mathrm{R}, \mathrm{E}$ & $\mathrm{R}, \mathrm{Sd}$ & $\mathrm{R}, \mathrm{Sd}, \mathrm{D}$ & 8 & & & \\
\hline 682 & 24 & $\mathrm{~W}$ & Ruschein & $\mathrm{R}$ & $\mathrm{R}$ & $\mathrm{Sd}$ & 5 & 1 & & \\
\hline 826 & 26 & $\mathrm{~W}$ & Laax & $\mathrm{R}$ & $\mathrm{R}$ & $\mathrm{Sd}, \mathrm{D}, \mathrm{I}$ & 14 & & & \\
\hline 680 & 27 & $\mathrm{M}$ & Chur & $\mathrm{R}$ & $\mathrm{Sd}$ & $\mathrm{D}, \mathrm{E}$ & 6 & & & \\
\hline 832 & 27 & $\mathrm{~W}$ & Chur & $\mathrm{R}$ & $\mathrm{R}$ & $\mathrm{Sd}, \mathrm{R}$ & 34 & & & \\
\hline 703 & 28 & $\mathrm{~W}$ & Zürich & $\mathrm{R}$ & $\mathrm{F}$ & $\mathrm{Sd}$ & 2 & & & $2 \mathrm{~F}$ \\
\hline 837 & 28 & $\mathrm{~W}$ & Zürich & $\mathrm{R}$ & $\mathrm{Sd}$ & $\mathrm{Sd}, \mathrm{D}$ & 3 & 4 & 2 & \\
\hline 1196 & 28 & $\mathrm{~W}$ & Chur & $\mathrm{R}, \mathrm{E}$ & $\mathrm{Sd}, \mathrm{D}$ & $\mathrm{Sd}, \mathrm{D}$ & 2 & & 3 & \\
\hline 977 & 29 & $\mathrm{~W}$ & Ilanz & $\mathrm{R}$ & $\mathrm{R}$ & $\mathrm{R}, \mathrm{Sd}$ & 8 & & & \\
\hline 674 & 31 & $\mathrm{~W}$ & Chur & $\mathrm{R}, \mathrm{Sd}$ & $\mathrm{R}, \mathrm{Sd}$ & $\begin{array}{c}\mathrm{Sd}, \mathrm{D}, \mathrm{R}, \\
\mathrm{F}, \mathrm{E}\end{array}$ & 19 & 1 & 2 & \\
\hline 721 & 31 & $\mathrm{~W}$ & Samedan & $\mathrm{R}$ & & $\mathrm{Sd}, \mathrm{D}, \mathrm{I}$ & 3 & & & \\
\hline 668 & 34 & $\mathrm{~W}$ & Haldenstein & $\mathrm{R}$ & & $\mathrm{Sd}$ & 5 & & & \\
\hline 683 & 41 & $\mathrm{M}$ & Scuol & $\mathrm{R}, \mathrm{Sd}, \mathrm{D}$ & & I & 9 & 2 & 4 & \\
\hline 1118 & 41 & $\mathrm{M}$ & Felsberg & $\mathrm{R}$ & $\mathrm{Sd}, \mathrm{D}$ & & 5 & & & \\
\hline 697 & 48 & $\mathrm{M}$ & Chur & $\mathrm{R}, \mathrm{Std}$ & $\mathrm{Sd}$ & $\mathrm{D}, \mathrm{I}$ & 6 & & & $1 \mathrm{I}$ \\
\hline 899 & 49 & $\mathrm{~W}$ & Ilanz & $\mathrm{R}$ & $\mathrm{Sd}$ & $\mathrm{D}, \mathrm{I}$ & 14 & & & \\
\hline 205 & 72 & $\mathrm{M}$ & Sursee & $\mathrm{R}$ & $\begin{array}{c}\text { Sd, D, } \\
\text { F, I }\end{array}$ & $\begin{array}{c}\mathrm{Sd}, \mathrm{D}, \mathrm{F}, \\
\mathrm{I}, \mathrm{R}\end{array}$ & 1 & & & \\
\hline 3222 & & W & & & & & 11 & & & \\
\hline 3284 & & & & & & & 5 & & & \\
\hline 3289 & & & & & & & 3 & & & \\
\hline 3299 & & $\mathrm{~W}$ & & & & & 3 & & & \\
\hline 3331 & & & & & & & 3 & 2 & & \\
\hline 3364 & & & & & & & 1 & 1 & & \\
\hline & & & & & & SMS: 297 & 214 & 67 & 11 & 5 \\
\hline
\end{tabular}

Tabelle 1: Sprachen der an der Datensammlung Teilnehmenden - Sprachen der SMS 
Will man alle Personen erfassen, die einen wie auch immer gestalteten Bezug zum Rätoromanischen aufweisen, ist die bisher besprochene Gruppe um zwölf Personen zu erweitern (cf. Tabelle 2). Die ergänzte Gruppe setzt sich folgendermassen zusammen:

- Drei Personen sind rätoromanischer Muttersprache, haben jedoch keine rätoromanischen SMS eingesandt.

- Sechs Personen, die das Rätoromanische nicht als ihre Muttersprache bezeichnen, verwenden es im privaten und/oder im öffentlichen Bereich. Nur eine dieser Personen nimmt ein Code-Switching ins Rätoromanische vor, das allerdings kurz und fehlerhaft ist (chè fas? für che fast? "was machst du?"), so dass ungewiss ist, ob die betreffende 17-jährige Teilnehmerin Rätoromanisch schreiben gelernt hat. ${ }^{4}$ Aus den Angaben zweier weiterer Personen gehen beschränkte Kompetenzen im Rätoromanischen hervor: Ein 18-jähriger gibt an, mit seiner Freundin rätoromanisch zu sprechen, während eine 21-jährige zum Code-Switching bemerkt: "Manchmal zum Spass Auschschnitte [sic!] aus irgendeiner andere Fremdsprachen [sic!], die ich eigentlich nicht beherrsche (wie Rätoromanisch...)". Die hierbei deklarierte Praxis wird allerdings mit den eingesandten SMS nicht bestätigt.

- Zwei Personen geben das Rätoromanische weder als Muttersprache noch als gesprochene Sprache an, verwenden jedoch Rätoromanisches in ihren SMS: die eine Person Gruss- und Dankesfloskeln in drei SMS, die andere eine Anrede (char 'Lieber') in einer SMS.

- Eine Person, die den Fragebogen nicht ausgefüllt hat, verfasst in ihrer einzigen eingesandten SMS den ersten Satz und die abschliessende Grussfloskel auf Rätoromanisch, während sie für den dazwischenliegenden, zwei Sätze umfassenden Kernteil der Nachricht das Deutsche verwendet.

\begin{tabular}{|c|c|c|c|c|c|c|c|c|c|c|}
\hline \multicolumn{7}{|c|}{$\begin{array}{l}\text { Personen mit Angaben zum Rätoromanischen und/oder } \\
\text { mit Code-Switching ins Rätoromanische }\end{array}$} & \multicolumn{4}{|c|}{ Anzahl SMS } \\
\hline 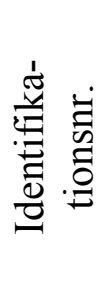 & $\stackrel{\Xi}{巳}$ & $\begin{array}{l}\frac{\vec{n}}{0} \\
\frac{0}{0} \\
\frac{0}{0} \\
0 \\
0 \\
0\end{array}$ & 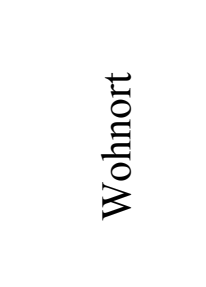 & 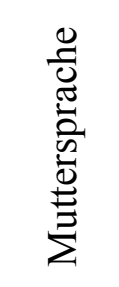 & 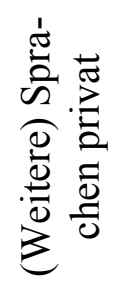 & 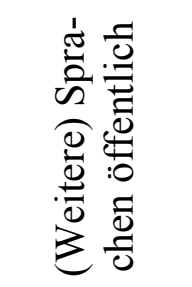 & 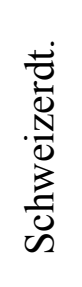 & $\begin{array}{l}\text { లే } \\
\stackrel{0}{0} \\
\stackrel{0}{0}\end{array}$ & 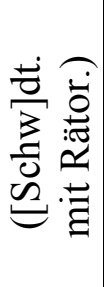 & $\frac{\mathscr{\Xi}}{\frac{D}{\Xi}}$ \\
\hline 760 & 12 & $\bar{W}$ & $\begin{array}{l}\text { Münsingen } \\
\text { (BE) }\end{array}$ & $\mathrm{Sd}$ & $\overline{F, R}$ & $\bar{F}$ & 24 & & & \\
\hline 566 & 16 & $\mathrm{~W}$ & $\begin{array}{l}\text { Dinhard } \\
(\mathrm{ZH})\end{array}$ & $\mathrm{Sd}$ & & $\mathrm{D}, \mathrm{F}, \mathrm{R}, \mathrm{E}$ & 133 & 5 & & $1 \mathrm{E}$ \\
\hline 373 & 17 & $\mathrm{~W}$ & $\begin{array}{l}\text { Sulgen } \\
\text { (TG) }\end{array}$ & $\mathrm{Sd}, \mathrm{R}$ & $\mathrm{Sd}$ & $\underset{\mathrm{E}}{\mathrm{D}, \mathrm{Sd}, \mathrm{F}}$ & 30 & & & \\
\hline 520 & 17 & $\mathrm{~W}$ & Zürich & $\mathrm{Sd}$ & $\mathrm{D}$ & $\mathrm{R}$ & 5 & & (1) & \\
\hline 630 & 18 & $\mathrm{M}$ & $\begin{array}{l}\text { Embrach } \\
(\mathrm{ZH})\end{array}$ & $\mathrm{Sd}$ & $\mathrm{F}, \mathrm{R}$ & & 2 & & & \\
\hline 1155 & 21 & $\mathrm{~W}$ & $\begin{array}{l}\text { Filisur } \\
\text { (GR) }\end{array}$ & $\mathrm{Sd}$ & $\begin{array}{l}\mathrm{D}, \mathrm{Sd}, \\
\mathrm{R}\end{array}$ & $\mathrm{D}, \mathrm{Sd}, \mathrm{I}, \mathrm{R}$ & 22 & & & \\
\hline 1202 & 21 & $\mathrm{M}$ & Zürich & $\mathrm{Sd}$ & $\mathrm{Sd}$ & $\mathrm{Sd}$ & 12 & & (1) & \\
\hline
\end{tabular}

\footnotetext{
${ }^{4}$ Für Scuol, den Hauptort des Unterengadins, sind jedoch dialektale Formen vom Typ has '(du) hast' (DRG s. avair, zu Punkt E 16), fas '(du) machst' und sas '(du) weisst' für unterengadinisches hast [af], fast [faf] und sast [saf] belegt.
} 


\begin{tabular}{|c|c|c|c|c|c|c|c|c|c|c|}
\hline \multicolumn{7}{|c|}{$\begin{array}{l}\text { Personen mit Angaben zum Rätoromanischen und/oder } \\
\text { mit Code-Switching ins Rätoromanische }\end{array}$} & \multicolumn{4}{|c|}{ Anzahl SMS } \\
\hline 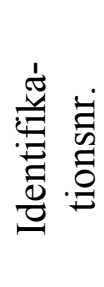 & 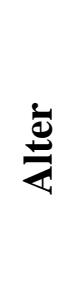 & $\begin{array}{l}\vec{t} \\
\frac{0}{0} \\
\frac{0}{0} \\
0 \\
0 \\
0 \\
0\end{array}$ & $\begin{array}{l}\frac{ \pm}{0} \\
\frac{1}{0} \\
\dot{0}\end{array}$ & 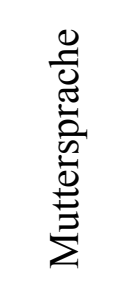 & 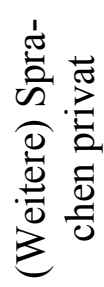 & 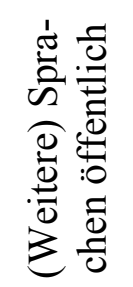 & 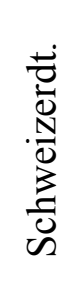 & 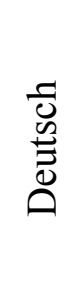 & 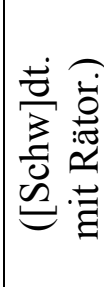 & 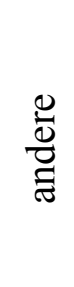 \\
\hline 439 & 24 & $\mathrm{~W}$ & & $\mathrm{Sd}, \mathrm{R}$ & $\mathrm{Sd}, \mathrm{R}$ & $\mathrm{D}$ & 1 & 3 & & \\
\hline 234 & 37 & $\mathrm{M}$ & $\begin{array}{l}\text { Rebstein } \\
\text { (SG) }\end{array}$ & $\mathrm{Sd}$ & & $\mathrm{R}, \mathrm{E}$ & 64 & 16 & & $7 \mathrm{E}$ \\
\hline 847 & 37 & $\mathrm{~W}$ & $\begin{array}{l}\text { Horgen } \\
(\mathrm{ZH})\end{array}$ & $\mathrm{Sd}$ & & $\mathrm{D}, \mathrm{E}$ & & 6 & (3) & \\
\hline 537 & 60 & W & $\begin{array}{l}\text { Mastrils } \\
\text { (GR) }\end{array}$ & $\mathrm{R}$ & Sd, I & $\mathrm{R}, \mathrm{F}, \mathrm{E}$ & 1 & 5 & & \\
\hline 2440 & & & & & & & & 1 & (1) & \\
\hline \multicolumn{7}{|c|}{ Total SMS: 338} & 294 & 36 & $(6)$ & 8 \\
\hline
\end{tabular}

Tabelle 2: Sprachen der an der Datensammlung Teilnehmenden - Sprachen der SMS

Möglicherweise verfügt keine dieser zwölf Personen über eine Kompetenz des Rätoromanischen, die eine unbefangene Verwendung dieser Sprache in der SMSKommunikation erlauben würde. Zu berücksichtigen ist auch, dass von den zehn Personen dieser Gruppe, deren Wohnort bekannt ist, zwei in Deutschbünden (Filisur ${ }^{5}$ und Mastrils) und acht ausserhalb Graubündens leben. Von diesem Gesamtprofil hebt sich dasjenige der Gruppe der Verfasserinnen und Verfasser rätoromanischer SMS deutlich ab: Von den 24 Personen mit Angaben zum Wohnort lebt die Hälfte im rätoromanischsprachigen Gebiet, ein Drittel (acht Personen) in Chur und Umgebung (eingeschlossen sind dabei die Nennungen von Felsberg und Haldenstein), während nur ein Sechstel (vier Personen) den Wohnsitz ausserhalb Graubündens hat. Bei den in Chur und Umgebung ansässigen Personen wird es sich zumindest teilweise um Mitarbeitende von kulturellen und Verwaltungsinstitutionen handeln, die auch in ihrem Beruf rätoromanisch schreiben. Aufgrund der genannten Verhältnisse ist davon auszugehen, dass die 30 Verfasserinnen und Verfasser rätoromanischer SMS ein Kontaktnetz haben, in dem sie das Rätoromanische eher einsetzen können als die zwölf Personen mit einem schwachen Bezug zum Rätoromanischen.

Bemerkenswert ist, dass die erwähnten zwölf Personen, die weitgehend (schweizer)deutsche SMS verschicken, zusammengenommen produktiver sind als die 30 rätoromanisch schreibenden Teilnehmenden: Während die zwölf Personen mit einem schwachen Bezug zum Rätoromanischen insgesamt 338 SMS verfasst haben, stammen von den 30 Benutzerinnen und Benutzern des Rätoromanischen nur 297 SMS, wobei die auf Rätoromanisch verfassten SMS 72\% aller von dieser Gruppe eingesandten SMS ausmachen. Die aktivste Person in der rätoromanisch schreibenden Gruppe hat interessanterweise viel mehr schweizerdeutsche als rätoromanische SMS geliefert (44 von 53). Die am zweithäufigsten Schreibende kommt auf die stattliche Anzahl von 34 - durchweg auf Rätoromanisch verfassten - SMS und steht damit nur hinter zwei Personen der anderen Gruppe zurück, die 139 bzw. 87 - grösstenteils auf Schweizerdeutsch verfasste - SMS zur Verfügung gestellt haben. Auch wenn die beiden

\footnotetext{
5 Filisur hatte 1880 ein gutes Drittel Rätoromanischsprachiger, 1941 waren es knapp über 10\% und im Jahr 2000 noch 7,3\% (cf. Furer 2005: 141). Seit Ende des 19. Jahrhunderts gilt Filisur als weitgehend germanisiert (www.filisur.ch $>$ Unser Dorf $>$ Geschichte, Stand 19.9.2011). Schule und Verwaltung sind deutsch (cf. Furer 1996: 131). 2009 wurde infolge der Fusion der Primarschule mit der Nachbargemeinde Bergün, wo traditionell Rätoromanisch unterrichtet wird, fakultativer Rätoromanischunterricht eingeführt.
} 
Teilgruppen zu klein sind, um weitergehende Schlüsse zu erlauben, weisen die genannten numerischen Verhältnisse darauf hin, dass sich der eingeschränkte Kommunikationsradius der Kleinsprache Rätoromanisch auch in diesem Bereich des Alltags bemerkbar macht.

Was die Angaben zu den Umgangssprachen betrifft, ist auf Folgendes hinzuweisen: Die beiden anschliessend an die Frage nach der Muttersprache gestellten Fragen zu weiteren Sprachen im privaten und öffentlichen Bereich wurden nicht von allen Teilnehmenden korrekt beantwortet. Verschiedene Personen, die das Rätoromanische als Muttersprache angaben, haben es auch als "weitere Sprache" in den Rubriken zu den Umgangssprachen aufgeführt, so dass die entsprechenden Spalten der Tabellen 1 und 2 kein aussagekräftiges Bild vermitteln. Einige Teilnehmende sind davon ausgegangen, dass sich die Verwendung der Muttersprache von selbst versteht, während andere ausdrücklich darauf hinweisen wollten, dass sie ihre Muttersprache in den beiden Bereichen bzw. nur in einem von diesen verwenden.

Aus den Angaben zu den Sprachen im privaten und öffentlichen Bereich sowie zu den in verschiedenen Sprachen verfassten SMS in Tabelle 1 geht das Bild eines von Zwei- und Mehrsprachigkeit geprägten Alltags hervor. Der in der Gruppe der rätoromanisch Schreibenden erhobene Anteil rätoromanischer SMS (214 von 297, d.h. 72\%) mag vor diesem Hintergrund als relativ hoch erscheinen. Bemerkenswert ist zudem, dass von den 30 Teilnehmenden nur elf auch SMS in anderen Sprachen eingesandt haben. Es ist also möglich, dass gewisse Personen bestrebt waren, besonders zur Vertretung des Rätoromanischen beizutragen, und deshalb vorwiegend oder ausschliesslich rätoromanische SMS zukommen liessen.

Die soziodemographischen Daten zu den rätoromanisch Schreibenden (cf. Tabelle 3) weisen darauf hin, dass die jüngeren Jahrgänge gut vertreten sind. Das gilt mehr noch für Personen mit einer höheren Bildung und mit einer Tätigkeit im Dienstleistungssektor sowie für weibliche Teilnehmende:

- Von den insgesamt 24 Personen, die den Fragebogen ausgefüllt haben, sind 16, also zwei Drittel, unter 30-jährig.

- Von den 15 Personen, die bis 28 Jahre alt sind, sind zehn Schülerinnen bzw. Studentinnen und Studenten. Auszubildende sind nicht vertreten, und nur eine Person ist im Gewerbe tätig.

- Von den 15 Personen, die über 25-jährig sind, haben zwölf, also vier Fünftel, einen Hochschulabschluss. Die anderen drei Personen dieser Gruppe haben eine Mittelschulausbildung (= Abitur) absolviert.

- Von den 14 Personen, die eine berufliche Tätigkeit angegeben haben, arbeiten 13 im Dienstleistungsbereich und eine im Gewerbe.

- Unter den 26 Personen, deren Geschlecht bekannt ist, befinden sich nur sechs Männer.

Angesichts dieser Verhältnisse kann das rätoromanische Subkorpus offensichtlich nicht als repräsentativ für die Gruppe der rätoromanisch schreibenden Mobiltelefon-Benutzerinnen und -Benutzer angesehen werden. 
$\mathrm{S} 1=$ Sekundarstufe I

S2 $=$ Sekundarstufe II
$\mathrm{M}^{6}=$ Mittelschulabschluss

$\mathrm{H}=$ Hochschulabschluss
$\mathrm{S}=$ Schüler/in, Student/in

$\mathrm{G}=$ Gewerbe

$\mathrm{D}=$ Dienstleistungsbereich

\begin{tabular}{|c|c|c|c|c|c|c|c|c|c|}
\hline \multicolumn{6}{|c|}{ Verfasserinnen und Verfasser rätoromanischer SMS } & \multicolumn{4}{|c|}{ Anzahl SMS } \\
\hline 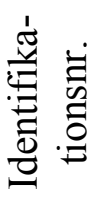 & 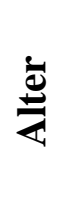 & 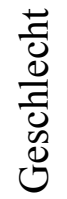 & Wohnort & Bildung & Beruf & 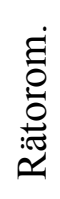 & 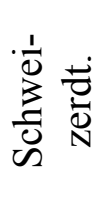 & 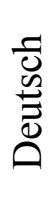 & 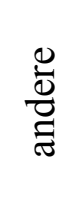 \\
\hline 1198 & 17 & $\mathrm{~W}$ & Pontresina & M & $\mathrm{S}$ & 6 & & & \\
\hline 764 & 18 & $\mathrm{~W}$ & Silvaplana & $\mathrm{M}$ & $\mathrm{S}$ & 5 & & & \\
\hline 667 & 19 & $\mathrm{~W}$ & Fex & $\mathrm{M}$ & $\mathrm{S}$ & 1 & & & \\
\hline 690 & 20 & $\mathrm{~W}$ & Fex & $\mathrm{M}$ & $\mathrm{S}$ & 9 & 44 & & \\
\hline 695 & 20 & $\mathrm{M}$ & Chur & $\mathrm{S} 2$ & $\mathrm{D}$ & 1 & 12 & & $2 \mathrm{E}$ \\
\hline 687 & 21 & $\mathrm{~W}$ & Zug & $\mathrm{M}$ & $\mathrm{S}$ & 16 & & & \\
\hline 733 & 21 & $\mathrm{~W}$ & Curaglia & S1 & $\mathrm{G}$ & 6 & & & \\
\hline 723 & 22 & W & Zuoz & $\mathrm{H}$ & $\mathrm{D}$ & 8 & & & \\
\hline 682 & 24 & $\mathrm{~W}$ & Ruschein & $\mathrm{M}$ & $\mathrm{S}$ & 5 & 1 & & \\
\hline 826 & 26 & $\mathrm{~W}$ & Laax & $\mathrm{H}$ & $\mathrm{S}$ & 14 & & & \\
\hline 680 & 27 & $\mathrm{M}$ & Chur & $\mathrm{H}$ & $\mathrm{D}$ & 6 & & & \\
\hline 832 & 27 & $\mathrm{~W}$ & Chur & $\mathrm{H}$ & $\mathrm{S}$ & 34 & & & \\
\hline 703 & 28 & $\mathrm{~W}$ & Zürich & $\mathrm{H}$ & $\mathrm{D}$ & 2 & & & $2 \mathrm{~F}$ \\
\hline 837 & 28 & $\mathrm{~W}$ & Zürich & $\mathrm{M}$ & $\mathrm{S}$ & 3 & 4 & 2 & \\
\hline 1196 & 28 & $\mathrm{~W}$ & Chur & $\mathrm{M}$ & $\mathrm{S}$ & 2 & & 3 & \\
\hline 977 & 29 & W & Ilanz & $\mathrm{H}$ & D & 8 & & & \\
\hline 674 & 31 & $\mathrm{~W}$ & Chur & $\mathrm{H}$ & $\mathrm{D}$ & 19 & 1 & 2 & \\
\hline 721 & 31 & $\mathrm{~W}$ & Samedan & $\mathrm{H}$ & $\mathrm{D}$ & 3 & & & \\
\hline 668 & 34 & $\mathrm{~W}$ & Haldenstein & $\mathrm{H}$ & $\mathrm{D}$ & 5 & & & \\
\hline 683 & 41 & $\mathrm{M}$ & Scuol & $\mathrm{H}$ & $\mathrm{D}$ & 9 & 2 & 4 & \\
\hline 1118 & 41 & $\mathrm{M}$ & Felsberg & $\mathrm{H}$ & $\mathrm{D}$ & 5 & & & \\
\hline 697 & 48 & $\mathrm{M}$ & Chur & $\mathrm{M}$ & $\mathrm{D}$ & 6 & & & $1 \mathrm{I}$ \\
\hline 899 & 49 & $\mathrm{~W}$ & Ilanz & $\mathrm{H}$ & $\mathrm{D}$ & 14 & & & \\
\hline 205 & 72 & $\mathrm{M}$ & Sursee & $\mathrm{H}$ & $\mathrm{D}$ & 1 & & & \\
\hline 3222 & & $\mathrm{~W}$ & & & & 11 & & & \\
\hline 3299 & & $\mathrm{~W}$ & & & & 3 & & & \\
\hline
\end{tabular}

Tabelle 3: Soziodemographische Daten der rätoromanisch Schreibenden

\section{Die rätoromanischen (Haupt-)Varietäten}

Das erste Raster für eine Differenzierung innerhalb des Variationsspektrums des Rätoromanischen liefert die Zuordnung zu den jeweiligen Schriftvarietäten. Der folgende Überblick über die Präsenz dieser Hauptvarietäten - teils, bei problematischen Zuordnungen, auch untergeordneter Varietäten - konfrontiert uns ein Stück weit mit der Architektur des Rätoromanischen in Bezug auf dessen räumliche und mediale (gesprochene vs. geschriebene) Variation:

- Die überwiegende Mehrzahl der rätoromanischen SMS (192 von 214) ist in regionalen Schriftvarietäten verfasst, wobei im Rahmen der regionalen Normen in gewissen

\footnotetext{
6 Die Angaben "Mittelschulabschluss" und "Hochschulabschluss" wurden nicht immer korrekt gewählt. Sie
} findet sich auch bei Personen, die diese Abschlüsse erst anstreben. 
Fällen dialektale Eigenheiten wiedergegeben werden. 22 SMS, d.h. 10\% der rätoromanischen SMS, sind dagegen in der seit 1982 entwickelten überregionalen Schriftsprache Rumantsch Grischun ${ }^{7}$ verfasst. Diese wird allerdings nur von einer Teilnehmenden in bedeutendem Umfang verwendet (18 SMS neben einer surselvischen und drei [schweizer]deutschen SMS, von denen eine auch Segmente in Rumantsch Grischun enthält). Die Teilnehmenden unserer Umfrage wurden in den regionalen Schriftsprachen alphabetisiert (als Alphabetisierungssprache in der Volksschule wird das Rumantsch Grischun erst seit 2007, in einem Teil der Gemeinden mit rätoromanischer Schule, verwendet). Wer heute Rumantsch Grischun schreibt, hat es also in einem institutionellen Kontext ausserhalb der obligatorischen Schulbildung erworben: auf Mittel- und Hochschulebene, im Rahmen einer Arbeit in den kantonalen Sprach- und Kulturorganisationen oder der Kantonsverwaltung oder im Rahmen der Weiterbildung für Lehrkräfte.

- Innerhalb der 192 SMS, die aufgrund der verwendeten Varietäten regional, teils auch lokal zugeordnet werden können, ist das Surselvische, das Rätoromanische des Vorderrheintals, mit zwei Dritteln aller regionalsprachlichen SMS überproportional vertreten, während die beiden Varietäten des Engadins und des Münstertals, das Puter (Oberengadin) und das Vallader (Unterengadin und Münstertal), die zusammen nicht einmal auf 30\% der regionalsprachlichen SMS kommen, untervertreten sein dürften. Schwach vertreten sind auch die Varietäten Mittelbündens, das Surmeirische (7 SMS) und das Sutselvische (1 SMS). Aufgrund der Ergebnisse der letzten Volkszählung zum Rätoromanischen als Haupt- und/oder Umgangssprache in den traditionell rätoromanischen Gemeinden lebt im Einzugsgebiet des Surselvischen etwas mehr als die Hälfte der Rätoromanisch Sprechenden ${ }^{8}$, während die Rätoromanisch Sprechenden des Engadins und des Münstertales ein gutes Drittel dieser Bevölkerungsgruppe ausmachen. ${ }^{9}$ Wie genau die Anteile der regionalen Varietäten in der gesamten Rätoromanisch sprechenden Bevölkerung, die $\mathrm{zu} 45 \%$ ausserhalb des traditionellen Sprachgebietes (in- und ausserhalb Graubündens) lebt, den genannten Anteilen entsprechen, ist allerdings ungewiss. Hier geht es lediglich um eine approximative Inbezugsetzung der Varietätenanteile in den SMS zur Präsenz der Varietäten in der Rätoromanisch sprechenden Bevölkerung.

- Alle 30 Verfasserinnen und Verfasser rätoromanischer SMS benutzen regionale Schriftvarietäten; von den vier Personen, die nur innerhalb (schweizer)deutscher SMS rätoromanisch schreiben, verwenden drei das Vallader und eine das Rumantsch Grischun. Unter den Teilnehmenden, die mehrere Schriftvarietäten verwenden, befinden sich vier, die ihre SMS in Rumantsch Grischun und in einer oder zwei

\footnotetext{
7 Als Grundlage für diese Ausgleichssprache wurden in erster Linie drei der fünf traditionellen Regionalschriftsprachen gewählt (Surselvisch, Surmeirisch und Vallader, cf. Schmid 1982). Innerhalb des damit gegebenen Variationsspektrums wurde nach dem Mehrheitsprinzip entschieden. In Fällen, in denen die genannten Regionalschriftsprachen keine ausreichende Entscheidungsgrundlage lieferten, wurden auch die beiden anderen Regionalschriftsprachen (Puter und Sutselvisch) sowie die dialektalen Verhältnisse berücksichtigt. Weitere Entscheidungskriterien waren die regionale Ausgewogenheit, die möglichst breite Verständlichkeit und die formale Transparenz (für weitere Grundinformationen zum Rumantsch Grischun cf. Gross 2004: 92-99). In der lexikalischen Semantik und in der Syntax, wo sich aufgrund divergierender regionaler Gebrauchsnormen beträchtliche Variationsspektren ergeben, kann die Standardisierung allerdings noch nicht als abgeschlossen gelten.

8 17'897 von 33'450 Personen, d.h. die Rätoromanisch Sprechenden der traditionell rätoromanischen Gemeinden der Bezirke Vorderrhein, Glenner und Imboden (Grünert et al. 2008: 48f.). Die rätoromanischen Gemeinden des Bezirks Imboden werden, mit der Ausnahme von Flims, aufgrund ihrer Mundarten als sutselvisch eingestuft, schriftlich verwenden sie jedoch traditionell das Surselvische.

9 11'772 von 33'450 Personen, d.h. die Rätoromanisch Sprechenden der traditionell rätoromanischen Gemeinden der Bezirke Münstertal, Inn und Maloja (Grünert et al. 2008: 45f.).
} 
regionalen Varietäten verfassen ${ }^{10}$, sowie zwei, die je in zwei regionalen Varietäten (Surselvisch und Puter bzw. Puter und Vallader) schreiben. Einen Sonderfall stellt die Person dar, die neben vier surselvischen SMS die einzige sutselvische SMS zur Verfügung gestellt hat. Letztere folgt nicht der sutselvischen Schriftnorm, sondern gibt nach den Prinzipien der surselvischen Orthographie den sutselvischen Dialekt der Gemeinde Domat/Ems wieder. ${ }^{11}$

\begin{tabular}{|c|c|c|c|c|c|c|c|c|c|c|}
\hline \multirow[b]{2}{*}{ 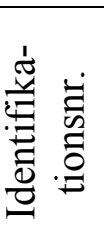 } & \multirow[b]{2}{*}{ 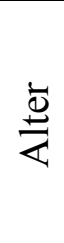 } & \multirow[b]{2}{*}{ 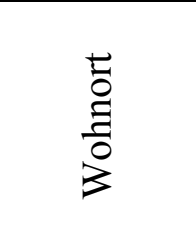 } & \multirow[b]{2}{*}{ 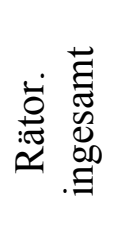 } & \multirow[b]{2}{*}{ 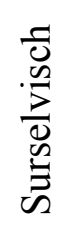 } & \multirow[b]{2}{*}{ 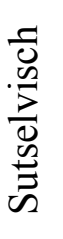 } & \multirow{2}{*}{ 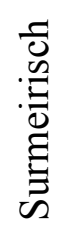 } & \multicolumn{2}{|c|}{ Engadinisch } & \multirow[b]{2}{*}{ 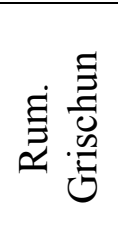 } & \multirow[b]{2}{*}{ 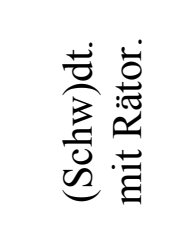 } \\
\hline & & & & & & & 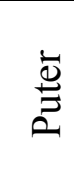 & 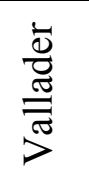 & & \\
\hline 520 & 17 & Zürich & & & & & & & & 1 Vallader \\
\hline 1198 & 17 & Pontresina & 6 & 4 & & & 2 & & & \\
\hline 764 & 18 & Silvaplana & 5 & & & & 5 & & & \\
\hline 667 & 19 & Fex & 1 & & & & 1 & & & \\
\hline 690 & 20 & Fex & 9 & & & & 9 & & & 1 Puter \\
\hline 695 & 20 & Chur & 1 & 1 & & & & & & \\
\hline 687 & 21 & Zug & 16 & 16 & & & & & & \\
\hline 733 & 21 & Curaglia & 6 & 6 & & & & & & \\
\hline 1202 & 21 & Zürich & & & & & & & & 1 Vallader \\
\hline 723 & 22 & Zuoz & 8 & & & & 5 & 3 & & \\
\hline 682 & 24 & Ruschein & 5 & 5 & & & & & & \\
\hline 826 & 26 & Laax & 14 & 10 & & & & 4 & & \\
\hline 680 & 27 & Chur & 6 & 6 & & & & & & \\
\hline 832 & 27 & Chur & 34 & 34 & & & & & & \\
\hline 703 & 28 & Zürich & 2 & & & & 2 & & & \\
\hline 837 & 28 & Zürich & 3 & & & & & 3 & & \\
\hline 1196 & 28 & Chur & 2 & & & & & 2 & & \\
\hline 977 & 29 & Ilanz & 8 & 8 & & & & & & \\
\hline 674 & 31 & Chur & 19 & 1 & & & & & 18 & 1 Rum. Gr \\
\hline 721 & 31 & Samedan & 3 & & & & & 3 & & \\
\hline 668 & 34 & Haldenst. & 5 & 5 & & & & & & \\
\hline 847 & 37 & Horgen & & & & & & & & 3 Vallader \\
\hline 683 & 41 & Scuol & 9 & & & & & 9 & & \\
\hline 1118 & 41 & Felsberg & 5 & & & 3 & & 1 & 1 & \\
\hline 697 & 48 & Chur & 6 & & & 4 & & & 2 & \\
\hline 899 & 49 & Ilanz & 14 & 14 & & & & & & \\
\hline 205 & 72 & Sursee & 1 & & & & 1 & & & \\
\hline 2440 & & & & & & & & & & $1 \mathrm{Rum} . \mathrm{Gr}$ \\
\hline 3222 & & & 11 & 10 & & & & & 1 & \\
\hline 3284 & & & 5 & 4 & 1 & & & & & \\
\hline
\end{tabular}

${ }^{10}$ Ausser der Person (Nr. 1118), die in der Tabelle mit drei Varietäten erscheint, ist eine Person zu erwähnen (Nr. 674), die im Fragebogen angibt, ausser den beiden im Korpus dokumentierten Varietäten, dem Rumantsch Grischun und dem Surselvischen, auch das Puter zu verwenden.

11 Die Gemeinde Domat/Ems liegt ausserhalb desjenigen Teils der Sutselva, für den die sutselvische Schriftsprache entwickelt wurde (die Regionen Schams, Domleschg und Heinzenberg; cf. Mani 1977: IX). Der Dialekt von Domat/Ems wird dem Sutselvischen zugeordnet (cf. DRG, Bd. 1: 24), die traditionelle Schriftvarietät ist jedoch das Surselvische. Wie unser Kommentar zu der im Emser Dialekt verfassten Textnachricht zeigt (siehe unten, Beispiel 4), sind die Abweichungen vom Schriftsurselvischen beträchtlich. 


\begin{tabular}{|c|c|c|c|c|c|c|c|c|c|c|}
\hline \multirow[b]{2}{*}{ 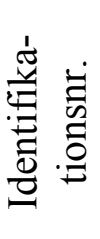 } & \multirow[b]{2}{*}{$\frac{\dot{\Phi}}{\frac{\bar{d}}{4}}$} & \multirow[b]{2}{*}{ 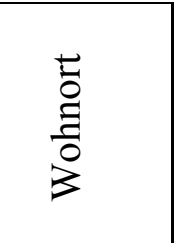 } & \multirow[b]{2}{*}{ 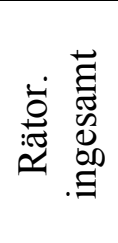 } & \multirow{2}{*}{ 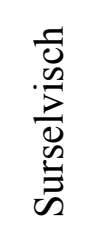 } & \multirow{2}{*}{ 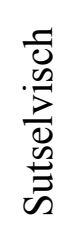 } & \multirow{2}{*}{ 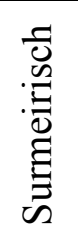 } & \multicolumn{2}{|c|}{ Engadinisch } & \multirow[b]{2}{*}{ 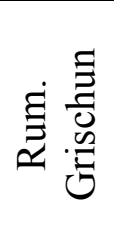 } & \multirow[b]{2}{*}{ 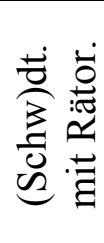 } \\
\hline & & & & & & & 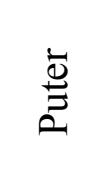 & $\frac{\overline{\bar{d}}}{\overline{\bar{J}}}$ & & \\
\hline 3289 & & & 3 & & & & 3 & & & \\
\hline 3299 & & & 3 & 3 & & & & & & \\
\hline 3331 & & & 3 & & & & & 3 & & \\
\hline 3364 & & & 1 & 1 & & & & & & \\
\hline \multicolumn{3}{|c|}{ Alle rätorom. SMS } & 214 & 128 & 1 & 7 & 28 & 28 & 22 & 8 \\
\hline \multicolumn{4}{|c|}{ Anteil in \% } & 59,8 & 0,5 & 3,3 & 13,1 & 13,1 & 10,3 & \\
\hline \multirow{2}{*}{\multicolumn{2}{|c|}{ SMS in reg }} & Varietäten & 192 & 128 & 1 & 7 & 28 & 28 & & \\
\hline & & \multicolumn{2}{|c|}{ Anteil in \% } & 66,7 & 0,5 & 3,6 & 14,6 & 14,6 & & \\
\hline
\end{tabular}

Tabelle 4: Rätoromanische Varietäten der SMS

\section{Sprechsprachliche (nicht sprachkontaktbedingte) Merkmale in den SMS}

Verschiedene Abweichungen von der jeweiligen durch die Schule vermittelten regionalen Schriftnorm ergeben sich dadurch, dass sich die Schreibenden an der sprechsprachlichen Norm orientieren und dazu neigen, im Geschriebenen Züge der Mündlichkeit wiederzugeben (Koch/Oesterreicher 2001: 585). Gewisse Erscheinungen betreffen die ganze Region einer Varietät. So wird im Vallader in bestimmten Positionen anstelle der stimmhaften alveolopalatalen Affrikate [ $\left.\mathrm{d}^{12}\right]^{12}$ der palatale Halbvokal [j] artikuliert. Von den drei Positionen, in denen die Affrikate [\$] gemäss der v.a. beim lauten Lesen realisierten Schulnorm des Valladers auftritt - im Anlaut vor Vokal (giuven ['dzuvən] 'jung'), im Inlaut intervokalisch (magöl [me'dъøl] 'Trinkglas') und im Inlaut postkonsonantisch (fingià [fin'dła] 'schon') - ist in der Sprechsprache nur Letztere erhalten. Im Anlaut vor Vokal und im Inlaut in intervokalischer Position wird dagegen [j] gesprochen (giuven ['juvən], magöl [me'jøl]). In einer sich an der Phonetik der Sprechsprache orientierenden Graphie wird nun statt 〈g〉 oder 〈gi〉 〈j〉 geschrieben, wenn die Aussprache [j] ist:

(1) Chau mam. Pudessast far ün plaschair a mai e trametter giò la staziun da chargiar la camera chi'd es aint in üna tas-china da palperi blaua jon ma stanza? [...] (ID 837, SMS 12509) $)^{13}$

Hallo, mam. Könntest du ${ }^{14}$ mir einen gefallen tun und das ladegerät der kamera, das in einer blauen papiertasche unten in meinem zimmer ist, herunterschicken ${ }^{15}$ ? [...]

\footnotetext{
${ }^{12}$ Zur nicht unumstrittenen Klassifikation dieses Lautes cf. Schmid (2010).

13 Alle zitierten SMS und Teile von SMS werden mit der Identifikationsnummer der Person (ID) und der SMSNummer versehen. Anschliessend folgt eine Übersetzung ins Deutsche, die nicht den Anspruch erhebt, stilistisch durchweg adäquat zu sein, sondern vor allem der Verständnishilfe dient. In der Übersetzung übernehmen wir die Klein- und Grossschreibung des Originals. Im Deutschen ist zwar die Kleinschreibung bei Substantiven, anders als im Rätoromanischen, markiert, sie ist jedoch eine in der SMS-Kommunikation oft wahrgenommene Möglichkeit, Tippaufwand einzusparen. Dass auch rätoromanisch Schreibende die Kleinschreibung als Ökonomieverfahren nutzen, wird aus der Kleinschreibung von Orts- und Personennamen sowie von Wörtern am Satzbeginn deutlich.

${ }^{14} \mathrm{Im}$ Deutschen könnte hier, ebenso wie im Rätoromanischen, das enklitische Subjektpronomen wegfallen. Wir verzichten jedoch in den Übersetzungen auf eine solche Ellipse, da diese im Deutschen einen anderen Stellenwert hat. Im Rätoromanischen ist die Auslassung des enklitischen Subjektpronomens der 2. Person in der Umgangssprache (im Engadin auch in der Schriftsprache) unmarkiert, während die entsprechende Auslassung im Deutschen stark sprechsprachlich markiert ist.

${ }^{15}$ Das Verb mit der lokalen Präzisierung, trametter giò 'herunterschicken', impliziert hier "aus dem Engadin ins Unterland schicken".
} 
Der Verfasser dieser SMS ist nicht konsequent, schreibt er doch für das Lokaladverb ('unten, hinunter, herunter') im ersten Fall gemäss der Schriftnorm des Valladers giò, im zweiten Fall dagegen gemäss der sprechsprachlichen phonetischen Realisierung jo, wobei er zusätzlich eine Agglutination der Präposition in 'in' vornimmt (jon, "unten in"). Damit setzt er sich in einer weiteren Hinsicht über die Schulnorm hinweg, nach der giò'n zu schreiben wäre.

Ein weiteres Beispiel bietet das umgangssprachliche Puter, das die Differenzierung zwischen $c u$ 'wie' (Frageadverb)/'als' (Vergleichskonjunktion) einerseits und scu 'wie' (Vergleichskonjunktion)/'als' (Appositionsmarker) andererseits aufgegeben hat (cf. Ganzoni 1977: 47f.). Scu übernimmt hier, zusätzlich zu den ihm ursprünglich eigenen Funktionen, die Funktionen von $c u$. In unserem Korpus ist nur die Verwendung von scu als Frageadverb dokumentiert:

(2) Chau tü,scu stest?est damaun darcho i'l tren per puntraschigna?salüd :-) (ID 1198, SMS 22048)

Hallo du,wie geht's dir?bist du morgen wieder im zug nach pontresina?gruss :-)

Die Aufgabe der Differenzierung zwischen der Partikel mit $c$ - und derjenigen mit $s c$ - (je nach Varietät $\mathrm{cu} / \mathrm{scu}$ oder $\mathrm{co} / \mathrm{sco}$ ) ist eine gemeinbündnerromanische Erscheinung, allerdings ist sie in den verschiedenen Varietäten unterschiedlich weit fortgeschritten. ${ }^{16}$ Während das Surmeirische nur noch scu kennt, ist die im Vallader und im Surselvischen auftretende Generalisierung von sco deutlich als sprechsprachlich markiert. In einer Zwischenposition zwischen diesen Extrempunkten situiert sich das Puter. In der gesprochenen Sprache ist hier generalisiertes scu unmarkiert, während es geschrieben weiterhin als ungrammatisch gilt. ${ }^{17} \mathrm{Zu}$ berücksichtigen ist bei dieser kontrastiven Analyse, dass die Partikel co/cu nur im Engadinischen (Vallader und Puter) auch als Konjunktion des Vergleichs dient, während sie in den übrigen Varietäten nur Frageadverb ist. Die Generalisierung im Surselvischen und Surmeirischen betrifft also lediglich die zusätzliche Verwendung von $s c o / s c u$ als Frageadverb, während sie im Vallader und Puter auch die Verwendung in Vergleichskonstruktionen mit dem Komparativ einschliesst (plü grond sco/pü grand scu statt plü grond co/pü grand cu, "grösser als"). Dieser Fall macht deutlich, wie wichtig es ist, bei der Beurteilung der sprachlichen Fakten die Besonderheiten der regionalen Normen zu berücksichtigen.

Markiert sprechsprachlich ist die im Surselvischen verbreitete Bezeichnung von Mitteln und Instrumenten (im weitesten Sinne 'Dinge, die einem Zweck dienen') mit Hilfe der Struktur "Demonstrativum + Präpositionalsyntagma", wobei dem Demonstrativum die Rolle des Determinatums zukommt und dem Präpositionalsyntagma diejenige des Determinans. Dies

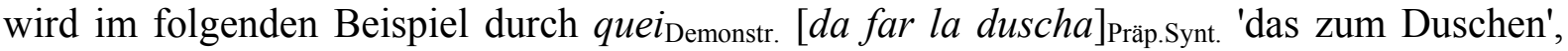
d.h. 'Duschmittel, Duschzeug' veranschaulicht, wo das Demonstrativum (quei 'das') eine unspezifische Referenz leistet, während das Präpositionalsyntagma präzisiert, für welche Tätigkeit (far la duscha 'duschen') die bezeichnete Sache bestimmt ist (Präp. $d a$ 'zu' zur Angabe des Zwecks):

\footnotetext{
16 Cf. die gemeinsame Behandlung von co und sco im $D R G$ (Bd. 4: 1-7).

17 Dass generalisiertes $s c u$ im umgangssprachlichen Puter schon lange eine Realität ist, lässt sich aus einer Passage in Reto Caratschs Satire La Renaschentscha dals Patagons ("Die Wiedergeburt der Patagonier") von 1949 schliessen, wo die von Puristen geforderte Unterscheidung zwischen $c u$ und $s c u$ aufs Korn genommen wird: "Scha qualchün s'incarognaiva da pcher cunter las reglas dal s-chet patagon, sch'el faiva pastrügls cul gerundi, tschavattaiva cunter il conjunctiv, confundaiva cu e scu, cuas e scuas, faiva nos specialist ün sagl tres l'ajer..." ("Wenn jemand hartnäckig gegen die Regeln des reinen Patagonischen sündigte, wenn er mit dem Gerundium ein Durcheinander anstellte, mit dem Konjunktiv pfuschte, cu und scu, cuas ['Schwänze'] und scuas ['Besen'] verwechselte, machte unser Spezialist einen Sprung durch die Luft...") (Caratsch 1983: 38).
} 
(3) [...] clar,hai schon priu cun mei igl uorden:-), mo quei da far la duscha hai emblidau....astgel lu ver in tec da tei? [...] (ID 832, SMS 19116)

[...] klar,habe die sachen schon mitgenommen:-),aber das duschzeug habe ich vergessen... darf ich dann ein wenig von dir haben? [...]

Was wie eine okkasionelle Umschreibung aussehen mag, ist tatsächlich ein in der Umgangssprache kodifiziertes Bezeichnungsmuster, das eine leichte Übertragung deutscher Bezeichnungen ins Rätoromanische ermöglicht. Eine Lehnübersetzung liegt allerdings nicht vor, verzichtet doch hier das gesprochene Surselvische auf eine Präzisierung des Determinatums, wie wir sie im Deutschen und bei den für das geschriebene Rätoromanische empfohlenen Lösungen vorfinden (cf. die beiden folgenden Tabellen). Da die Periphrase mit dem Demonstrativum bisher in der Literatur nicht beschrieben worden ist, gehen wir aufgrund von Beispielen, die wir bei Sprecherinnen und Sprechern gesammelt haben, ausführlicher auf sie ein. Bemerkenswert ist die Differenzierung in dieser Periphrase zwischen dem in Beispiel (3) veranschaulichten generischen quei 'das'18 und dem individualisierenden quel 'der'. Generisches quei wird bei Stoff- und Kollektivbezeichnungen verwendet:

\begin{tabular}{|l|l|l|}
\hline $\begin{array}{l}\text { Rätor. sprechsprachlich } \\
\text { (Surselvisch) }\end{array}$ & $\begin{array}{l}\text { Rätor. schriftsprachlich } \\
\text { (Rumantsch Grischun'19) }\end{array}$ & Deutsch \\
\hline quei da far la duscha & $\begin{array}{l}\text { savun liquid } \\
\text { (savun 'Seife') }\end{array}$ & $\begin{array}{l}\text { Duschzeug, Duschmittel, } \\
\text { Flüssigseife }\end{array}$ \\
\hline $\begin{array}{l}\text { quei da far la barba } \\
\text { (far la barba } \text { 'rasieren') }\end{array}$ & $\begin{array}{l}\text { stgima/crema/savun da far } \\
\text { la barba }\end{array}$ & $\begin{array}{l}\text { Rasierschaum/-crème/ } \\
\text {-seife }\end{array}$ \\
\hline $\begin{array}{l}\text { quei da far la barba } \\
\text { (urden/rauba da far la barba } \text { rauba 'Zeug') }\end{array}$ & Rasierzeug \\
\hline $\begin{array}{l}\text { quei da dar si la fatscha } \\
\text { (dar si 'auftragen' [dar 'geben' } \\
+ \text { si 'auf']; fatscha 'Gesicht') }\end{array}$ & bellet, schminca & Schminke \\
\hline $\begin{array}{l}\text { quei da lavar giu } \\
\text { (lavar 'waschen' + giu '[her- } \\
\text { /hin-]ab', nach dt. abwaschen) }\end{array}$ & $\begin{array}{l}\text { med da lavar giu } \\
\text { (med 'Mittel'), } \\
\text { detergent }\end{array}$ & Abwaschmittel \\
\hline $\begin{array}{l}\text { quei da lavar ils mauns } \\
\text { (mauns 'Hände') }\end{array}$ & savun liquid & Flüssigseife für die Hände \\
\hline quei da schubergiar & med/product da nettegiar & Reinigungsmittel \\
\hline $\begin{array}{l}\text { quei da ligiar si } \\
\text { (ligiar 'binden', ligiar si } \\
\text { 'verbinden') }\end{array}$ & $\begin{array}{l}\text { material da faschar } \\
\text { (faschar 'verbinden') }\end{array}$ & Verbandzeug, -material \\
\hline $\begin{array}{l}\text { quei da pinar il velo } \\
\text { (pinar 'flicken') }\end{array}$ & utensils da reparar il velo \\
\hline $\begin{array}{l}\text { quei da magliar } \\
\text { vivandas, victualias, } \\
\text { mangiativas }\end{array}$ & Fahrrad-Reparaturzeug \\
\hline
\end{tabular}

Tabelle 5: Periphrasen mit quei bei Stoff- und Kollektivbezeichnungen

Das maskuline Demonstrativum quel wird dagegen bei Individualbezeichnungen gesetzt. Dabei handelt es sich vor allem um Bezeichnungen einzelner solider Instrumente:

18 Zum Status der in der traditionellen Grammatik als neutral bezeichneten Formen cf. Wunderli (1993: 156).

19 Nach der lexikalischen Datenbank Pledari grond. 


\begin{tabular}{|c|c|c|}
\hline $\begin{array}{l}\text { Rätor. sprechsprachlich } \\
\text { (Surselvisch) }\end{array}$ & $\begin{array}{l}\text { Rätor. schriftsprachlich } \\
\text { (Rumantsch Grischun) }\end{array}$ & Deutsch \\
\hline $\begin{array}{l}\text { quel da far giu la pelletscha } \\
\text { (far giu 'ab-/wegmachen', } \\
\text { pelletscha 'Schale') }\end{array}$ & $\begin{array}{l}\text { spaletschader } \\
\text { (zu spaletschar '[ab]schä- } \\
\text { len') }\end{array}$ & (Spar-)Schäler \\
\hline $\begin{array}{l}\text { quel da far la barba } \\
\text { (eher für 'Handrasierer') }\end{array}$ & $\begin{array}{l}\text { rasuir (electric), } \\
\text { apparat da far la barba }\end{array}$ & Rasierer, Rasierapparat \\
\hline $\begin{array}{l}\text { quel da far giu la pelegna } \\
\text { (pelegna 'Körperhaar') }\end{array}$ & rasuir da damas & Cosmetic-shaver \\
\hline quel da cargar & & Ladegerät \\
\hline quel da cargar & $\begin{array}{l}\text { bloc d'alimentaziun, } \\
\text { alimentader da current }\end{array}$ & Netzgerät \\
\hline quel da cargar il natel & chargiatelefonin & $\begin{array}{l}\text { Ladegerät für das } \\
\text { Mobiltelefon }\end{array}$ \\
\hline quel da cargar (las) batterias & $\begin{array}{l}\text { chargiader da battarias, } \\
\text { chargiabattarias }\end{array}$ & Batterieladegerät \\
\hline quel da marcar & marcader luminus & Marker, Leuchtstift \\
\hline
\end{tabular}

Tabelle 6: Periphrasen mit quel bei Individualbezeichnungen

Individualbezeichnungen können allerdings auch in Bezug auf unzählbare Referenten (Stoffe) vorgenommen werden, dann nämlich, wenn eine Selektion zwischen einer Mehrzahl solcher Referenten stattfindet. So heisst zwar das Abwaschmittel generell quei da lavar giu. Wenn jedoch das Abwaschmittel aus einer Klasse von Mitteln ausgesondert wird - um eine konkrete Alltagssituation zu nennen: wenn man z.B. um das Abwaschmittel und nicht um den daneben stehenden Behälter mit der Flüssigseife für die Hände bittet - sagt man quel da lavar giu und unterscheidet dieses somit von quel da lavar ils mauns.

Im Engadinischen wird neben dem hier veranschaulichten Bezeichnungsmuster mit dem Demonstrativum (nur mit individualisierendem quel: quel da/per ${ }^{20}$ far giò la pletscha '[Spar-] Schäler', quel da/per chargiar il telefonin 'Ladegerät für das Mobiltelefon') häufiger die Formel "Nomen (Determinatum) + Präpositionalsyntagma (Determinans)" verwendet, wobei das Nomen oft eine sehr weit gefasste Klassenbezeichnung ist, wie roba 'Sache' oder s-cherp, guaffen 'Ding, Gerät' (roba da/per far la duscha 'Duschmittel', s-cherp da/per chargiar las battarias 'Batterieladegerät'). Einen Beleg für eine Bezeichnung im Engadinischen liefert das obige Beispiel (1), wo, wie in den Fügungen in der mittleren Spalte der Tabellen 5 und 6, eine stärker einschränkende Klassenbezeichnung verwendet wird: staziun da chargiar la camera 'Ladegerät für die Kamera'. Dieser weniger sprechsprachlich charakterisierte Ausdruck ist in unserem SMS-Korpus eher erstaunlich.

Die Periphrasen zur Bezeichnung von Mitteln und Instrumenten in den beiden grösseren Regionen Romanischbündens unterscheiden sich nicht nur hinsichtlich ihrer Komponenten, sondern auch hinsichtlich ihres Status: Während die surselvischen Periphrasen mit quei da... und quel da ... in der sprechsprachlichen Norm als Bezeichnungen für die genannten Denotate verankert sind, gelten die weniger gefestigten engadinischen Periphrasen eher als Ersatzlösungen für zutreffendere Bezeichnungen (z.B. von Verben abgeleitete nomina agentis wie spletschader für '[Spar-]Schäler' oder chargiader für 'Ladegerät').

Neben sprechsprachlichen Erscheinungen, die regionale Varietäten betreffen, sind auch lokal oder mikroregional eingegrenzte mundartliche Phänomene bezeugt. Unter den SMS, die aus dem Gebiet stammen, in dem traditionell die surselvische Schriftsprache verwendet wird,

${ }^{20}$ Neben der Präposition $d a$ ist auch die Präposition per möglich, die ausdrücklicher der Zweckbezeichnung dient. Die Beispiele zum Engadinischen sind in Vallader (Unterengadinisch) wiedergegeben. 
finden sich folgende Belege für Mundarten: eine SMS, die weitgehend im Dialekt von Domat/Ems verfasst ist (4), eine SMS, die weitgehend im Dialekt des Tavetschs (Val Tujetsch) verfasst ist (5), sechs SMS, die mit Elementen des Tavetscher Dialekts durchsetzt sind (daraus Beispiel 6), und eine SMS, in der lediglich ein Wort eine für den Dialekt von Brigels/Breil charakteristische Lautung aufweist (7). Unter den SMS aus dem Einzugsgebiet des Valladers enthält ein Text eine lautliche und eine morphosyntaktische Besonderheit des Jauers, des Dialekts des Münstertals (8).

(4) car padreng! giavisch cun retard ong tut bian sin anniversare! bunga sanadad a glec! vegn proximamein ongaga ingaga sperasvéi! cars salids è di rest dalla famiglia! tiu figliol (ID 3284, SMS 7967)

lieber pate! wünsche mit verspätung noch alles gute zum geburtstag! gute gesundheit und glück! komme demnächst noch einmal vorbei! liebe grüsse auch vom rest der familie! dein patensohn ${ }^{21}$

(5) So té quaki?. Vo quai? Muahaha tgei marscha w-end? Loto u? Meh pupsi da adatg da te nevetg! Ct [= carezel te] bffenl [= betschel fetg ferm, weitere Dekodierung unklar] muah (ID 3364, SMS 14013)

So du quaki? Geht das? Muahaha was läuft [am] w-end? Lotto nicht wahr? Meh pupsi pass auf dich auf nicht wahr! Hdl [= hab dich lieb] küss dich ganz fest [weitere Dekodierung unklar]

(6) heho e bien di cara durmiu bein e pudiu lavar?ju schon.nuvas?ju betg.bi de e fai mu empau.tau tau (ID 733, SMS 9264)

heho und guten tag liebe gut geschlafen und aufstehen können?ich schon.neues?ich nicht.schönen tag und mach nur ein wenig.t[sch]au t[sch]au

(7) Bialas fotis:-)!so,fertic cun far patgific...bi sm [= suentermiezdi],e tochen gloiti.bunis (ID 832, SMS 11180)

Schöne fotos:-)!so,fertig mit gemächlich machen...schönen nm [= nachmittag],und bis bald.küsschen

(8) Aha..hai poßi schlö eir.. Lura jarasch las 4sül tren e esch mez las 5 qua..odr cheu vegn jo z. E fain cajò..co cha tù vosch.. (ID 3331, SMS 10393)

Aha..ja kann ich sonst auch.. Dann gehst du um 4auf den zug und bist um halb 5 hier..oder ich komme nach z[ürich] hinunter. Und wir machen [es] dort unten..wie du willst..

In den zitierten SMS lassen sich folgende dialektal begründete Abweichungen vom geschriebenen Surselvischen beobachten:

- zu (4): padreng vs. padrin 'Pate' (HWR s. padrin), giavisch (1. Pers. ohne Endungsmorphem, cf. dazu Bsp. im Mundarttext aus Domat/Ems in Schorta 1946) vs. giavischel '(ich) wünsche', ong vs. aunc 'noch' (DRG s. auncha, C 9222), bian vs. bien 'gut' m. (DRG s. bun, C 92), bunga vs. buna 'gut' f. (DRG s. bun, C 92), glec [glek] vs. cletg [kletc] 'Glück' (DRG s. cletg, C 92 vs. S), ongaga ingaga vs. aunc inaga 'noch

\footnotetext{
21 Standarddeutsches Pate und Patensohn, das wir in der zur Verständnishilfe angeführten Übersetzung verwenden, wird von Deutschschweizerinnen und Deutschschweizern in der privaten Korrespondenz - umso mehr in der durch einen informellen Stil geprägten SMS-Kommunikation - nicht gebraucht. Im deutschen Teil des Schweizer SMS-Korpus sind nur Götti (in drei in Schweizerdeutsch und in vier in Standarddeutsch verfassten SMS) und Göttibueb (in einer in Schweizerdeutsch verfassten SMS) bzw. das phonetisch angepasste Göttibub (in drei in Standarddeutsch verfassten SMS) belegt.

${ }^{22}$ Hier und in der Folge: Aufnahmepunkte und -gebiete des $D R G(\mathrm{C} 92=$ Domat/Ems, $\mathrm{S}=$ Surselva, $\mathrm{S} 70-74=$ Val Tujetsch, S 7 = Val Tujetsch und Val Medel, S 1-6 = die Surselva unter Ausschluss der Val Tujetsch und der Val Medel).
} 
einmal' (im Emser Dialekt ongaga 'nochmal' + ingaga 'einmal'; zu ingaga vs. inaga cf. Rupp 1963: 66f.), sperasvéi vs. sperasvi 'vorbei' (HWR s. sperasvi und Rupp 1963: 12f.). Nicht dem Emser Dialekt entspricht das Adverbialsuffix -mein in proximamein 'demnächst', an dessen Stelle -megn [men] zu setzen wäre (cf. DRG, Registerband: 91, s. -mente, C 9). Diese Inkonsequenz zeigt, dass Schreibroutinen, die sich im Standarddeutschen verfestigt haben, beim spontanen Mundartschreiben durchdringen, ein Phänomen, das auch beim Mundartschreiben in der Deutschschweiz bemerkt wird (cf. Dürscheid/Spitzmüller 2006: 23).

- zu (5): té [te] vs. $t i$ 'du' (Caduff 1952: 39), vo vs. va 'geht' (DRG s. ir, S 70-74), quai (markierte Graphie für [kwai]) vs. quei (Graphie, der verschiedene Realisierungen zugeordnet sind: [kwعi]/[kwoi]/[kwai]/[kwoi]) 'das' (cf. DRG s. mai 'mich', mit demselben Dipthong, S 7 vs. S 1-6), da vs. dai 'gib!' (DRG s. dar, S 72), te [t $\varepsilon$ ] vs. tei [tzi]/[toi]/[tai]/[toi] 'dich' (cf. Caduff 1952: 39), nevetg vs. nevé 'nicht wahr'. Die Graphie tgei 'was' lässt sich nicht eindeutig zuordnen, sie ist schriftsurselvisch und gibt gleichzeitig eine dialektal belegte Variante wieder (Caduff 1952: 39). Eindeutig dialektal wäre die Graphie tge (DRG s. che, S 71 sowie Widmer 1963: 187).

- zu (6): ju vs. jeu 'ich' (DRG s. eu, S 70-74), betg [bet6] vs. buc(a) 'nicht' (DRG s. brich [a], S 7), de vs. $d i$ 'Tag' (DRG s. $d i, \mathrm{~S} 7), m u$ vs. $m o$ 'nur' (DRG s. $m a$, S 71). Die SMS beginnt nicht im Tavetscher Dialekt, sondern im Schriftsurselvischen. Eindeutig nicht dialektal sind allerdings im ersten Satz nur $d i$ 'Tag' und cara 'Liebe' (Person), an deren Stelle man in der Mundart de und tgera (DRG s. char, S 70-74) setzen würde.

- zu (7): gloiti ['gloiti] vs. gleiti ['gleiti]/['gloiti]/['gleiti] (DRG s. gleiti, S 26 vs. die übrigen Punkte in S). Nicht dem Brigelser Dialekt entspricht biala 'schön' f., an dessen Stelle bela ['bele] zu setzen wäre (DRG s. bel, S 26). Der Rest dieser kurzen SMS würde in Mundartgraphie nicht vom Schriftsurselvischen abweichen.

- zu (8): poßi (verkürzte Graphie für possi) vs. possa 'kann ich', d.h. poss 'kann' (1. Person) + enklitisches Pronomen der 1. Person, das in der Norm des Valladers $-a$ [e] lautet, im Dialekt des Münstertals dagegen - $i$. Innerhalb des Einzugsgebietes des Valladers teilt das Münstertalische diesen Zug mit der Mundart von Zernez, die an das Oberengadinische (Puter) grenzt, wo das enklitische Pronomen der 1. Person ebenfalls $-i$ lautet. Eindeutig als Münstertalisch lässt sich der Text aufgrund des Ausdrucks mez las tschinch ("halb fünf") identifizieren, welcher unterengadinischem las quatter e mez (wörtl. "vier und [eine] halb[e Stunde]") gegenübersteht. Nicht münstertalisch wiedergegeben ist bemerkenswerterweise das Subjektpronomen der 1. Person, jau, dem die Varietät des Münstertals ihren Namen, jauer, verdankt. Stattdessen steht gemäss der Norm des Valladers eu 'ich' (allerdings, eventuell wegen nicht verfügbarem Apostroph, an die Konjunktion $c h[a]$ agglutiniert: $c h e u$ statt $c h ' e u$ 'dass ich').

\section{$5 \quad$ Sprachkontaktbedingte Erscheinungen}

In den SMS finden sich zahlreiche Lehnwörter aus dem (Schweizer-)Deutschen, die für das gesprochene Rätoromanische charakteristisch sind, während die von der Schule vermittelte Schriftnorm deren Gebrauch untersagt bzw. puristische Ersatzwörter oder Ersatzausdrücke vorschreibt. Diese Germanismen bilden, neben den im vorhergehenden Kapitel veranschaulichten Zügen der Mündlichkeit, eine weitere Komponente, die zur sprechsprachlichen Gestaltung der rätoromanischen SMS-Kommunikation beiträgt: 
(9) Nus mein grad ord savognin. cs a (ID 668, SMS 7777, Surselvisch ${ }^{23}$ )

Wir verlassen gerade savognin. $\lg$ a

(10) Ciao tü,po esser cha stefi nu po gnir hoz saira,perche chal pass es serro.ma forsa evrane $e b a$ auncha. (ID 690, SMS 23467, Puter)

Hallo du,kann sein, dass stefi heute abend nicht kommen kann,weil der pass geschlossen ist.aber vielleicht öffnen sie eben noch.

(11) Quei ei prima aschia. Tochen damaun, salids lucrezia ${ }^{24}$ (ID 899, SMS 13170, Surselvisch)

Das ist prima so. Bis morgen, grüsse lucrezia

Verbreitet sind auch Lehnübersetzungen (vgl. 12-14, aber auch das obige Beispiel 10, wo hoz saira deutschem heute Abend folgt, während die rätoromanische Norm quista saira, mit dem Demonstrativum quista anstelle des Zeitadverbs hoz, vorsehen würde) sowie Lehnbedeutungen (14/15).

(12) Hallo Jeannine, co hast? Meglder? Speresch... [...] (ID 1196, SMS 21993, Vallader) Hallo Jeannine, wie geht's dir? Besser? Ich hoffe... [...]

(13) Bonsoir lorna, level mirar co ti hagies e dumandar cu ti mondies giu cuera margis [...] (ID 899, SMS 15678, Surselvisch)

Bonsoir lorna, wollte schauen, wie's dir geht und fragen, wann du am dienstag nach chur fährst. [...]

(14) [...] mon schon sin quart vargau per s'endrizzar. [...] (ID 832, SMS 19116, Surselvisch)

[...] ich gehe schon auf Viertel nach hin, um mich einzurichten. [...]

(15) $[\ldots]$ e vus,giu ina biala fin d'jamna?ina sera da giugs tuna super! [...] (ID 832, SMS 11176, Surselvisch)

[...] und ihr,ein schönes wochenende gehabt?ein spielabend tönt super! [...]

Die von Sprecherinnen und Sprechern jüngeren und mittleren Alters verwendete Lehnübersetzung co hast? [ko af] ("wie geht's dir"), nach schweizerdeutschem wie hesch es? (wörtl. wie hast du es?), wird von älteren Personen bisweilen mit der Gegenfrage co? asch? co? dutsch? [ko af ko dut]] ("wie? bitter? wie? süss?") oder mit der Antwort asch e dutsch [af e duff] ("bitter und süss") quittiert. Mit einem Homonymiespiel, in dem [af] 'bitter' intendiertem [af] 'hast (du)' gegenübergestellt wird, gibt man hier vor, die sich an das Schweizerdeutsche anlehnende Frage nach dem Befinden nicht zu verstehen. Darauf hinzuweisen ist, dass sich rätoromanisches co hast? gegenüber dem schweizerdeutschen Vorbild wie hesch es? durch das Fehlen des Objektpronomens auszeichnet. In dem in Beispiel (12) veranschaulichten Vallader ist diese Leerstelle zwingend, da für die 3. Person kein neutrales Objektklitikon verfügbar ist ( $i$ 'es' hat ausschliesslich Subjektfunktion). Als neutrales Objekt kann in dieser Varietät nur das Demonstrativum quai 'das' gesetzt werden, das eine konkretere Referenz impliziert und daher nicht adäquat wäre. Im Surselvischen tritt zwar ei 'es' bisweilen als Objektpronomen auf, häufig wird aber das neutrale Objekt mit generischer Referenz nicht mit einem Pronomen bezeichnet (cf. Spescha 1989: 336). So lautet die Lehnübersetzung auch im Surselvischen co has? bzw. in Beispiel (13) in indirekter Form co ti hagies ("wie es dir gehe"), ohne Objektpronomen. Was die Ellipse des Subjektpronomens der

\footnotetext{
$23 \mathrm{Zu}$ den in den Beispielen (9) und (10) veranschaulichten Adverbien cf. Solèr (1999a: 96f.).

Da die diatopischen Varietäten von nun an nicht mehr thematisiert werden, geben wir bei den Zitaten nach der Identifikationsnr. und der SMS-Nr. jeweils die Zugehörigkeit zur regionalen Varietät an.

24 Alle Namen wurden anonymisiert.
} 
2. Person bei Enklise betrifft (in der direkten Frage co hast?/co has?), so ist diese im Rätoromanischen, wie im Schweizerdeutschen, sehr verbreitet (cf. dazu unten in Kap. 6).

In Beispiel (14) gibt das Syntagma quart vargau deutsches Viertel nach, das nicht auf eine bestimmte numerisch bezeichnete Stunde bezogen ist, wieder. Die alternative, nicht durch den Sprachkontakt mit dem Deutschen bedingte Bezeichnung, l'ina ed in quart ("Viertel nach eins"), las duas ed in quart ("Viertel nach zwei") etc. erlaubt keine derartige generische - in diesem Fall aus Ökonomie verkürzte - Bezeichnung des ersten Viertels einer Stunde. Bei der Präposition sin, die quart vargau in Beispiel (14) vorausgeht, liegt eine in der Umgangssprache integrierte Lehnübersetzung vor: Die Lokalpräposition sin 'auf' wird ebenso wie die entsprechende deutsche Präposition auf für die Bezeichnung der zeitlichen Annäherung verwendet. ${ }^{25}$ Eine weitere Lehnübersetzung belegt Beispiel (15): tunar 'tönen (Klänge von sich geben)' hat unter dem Einfluss des deutschen tönen die zusätzliche Bedeutung 'einen Eindruck machen' erhalten.

Neben dem in der Umgangssprache integrierten Lehngut (cf. die bisher erläuterten Fälle, aber auch im folgenden Beispiel [16] benna, aus schwdt. Benne 'Karren', übertragen für 'Modem') sei Wortgut aus dem Deutschen erwähnt, dessen Gebrauch zwar plausibel ist, das aber trotzdem nicht als integriertes Lehngut gelten kann (festnetz in Beispiel 16, kraftraum in Beispiel 17). Es handelt sich dabei um Bezeichnungen, zu denen Rätoromanisch Sprechende nicht ohne Weiteres Äquivalente in ihrer Sprache bereit haben, so dass sich der Wechsel zum Deutschen ein Stück weit aufdrängt. ${ }^{26}$ Unumgänglich ist im Kontext in Beispiel (16) der Internationalismus skype, von dem allerdings eine deutsche Ableitung, skypen, in die rätoromanische Periphrase "far 'machen' + mask. Artikel $i l+\mathrm{dt}$. Infinitiv"27 aufgenommen ist. Diese Struktur, die ein fester Bestandteil der Umgangssprache ist, dient der Überbrückung lexikalischer Lücken bei der Bezeichnung von Handlungen.

(16) donat, mia benna funcziunescha insumma buca pli dad ir egl internet. savein buca far il skypen! has festnetz enzanua? cs (ID 3284, SMS 7966, Surselvisch) donat, meine benne, um ins internet zu gehen, funktioniert heute überhaupt nicht. können wir nicht skypen! hast du irgendwo festnetz? lg

(17) Eau sun uoßa güsta gnida a chesa, e zieva stögli ir illa clinica.. Vest hoz al kraftraum? [...] (ID 764, SMS 9862, Puter)

Ich bin jetzt gerade nach hause gekommen, und nachher muss ich in die klinik gehen.. Gehst du heute in den kraftraum? [...]

Weiter finden wir ein schweizerdeutsches Zitat - den mit der Läuferin Anita Weyermann assoziierten Satz "Gring abe u seckle!" ("Kopf tief und rennen!") (18) - sowie Code-

\footnotetext{
25 Zur Lehnsemantik bei Präpositionen cf. Solèr (1998: 157).

26 Zur Selbstverständlichkeit der Anleihen beim Deutschen cf. Solèr (2010: 415f.): "Contrairement à beaucoup de communautés plurilingues, la compétence linguistique, tant du romanche que de l'allemand, et le thème du discours n'influencent pas le choix linguistique. Au cas déficitaire, on emprunte à tous niveaux (phonétique, morphologique, lexique et métaphorique) et applique la voie transcodique, le "Code mixing". Dans ces cas, il s'agit normalement déjà d'un domaine allemand en distribution complémentaire, diglossique, comme la formation professionnelle supérieure, les entreprises dépassant la région étroite et même les loisirs (tv, cinéma, musique, magazines etc.)."

Cf. in diesem Zusammenhang auch Solèr (1999a: 104f.): "Für zweisprachige Menschen, die über das Inventar zweier Sprachen (teilweise komplementär) verfügen, scheinen die definierten Sprachgrenzen ein kleineres Hindernis zu sein als eine stilistisch unpassende Variante. Die historische und definitorische Sprachgrenze, bzw. Zuteilung des Materials zu einer oder zur anderen Sprache, wird in der täglichen Kommunikationssituation gegenüber dem aktuellen kollektiven Wissen um Zeichen kaum beachtet [...]."

27 Cf. Solèr (1998a: 158). Während im Surselvischen die Periphrase far il skypen üblich ist, bildet das Engadinische ein Verb, indem es entlehntes skype mit dem Verbalmorphem verbindet: skypar [skai'par] (Vallader)/skyper [skai'per] (Puter) 'skypen' (zu diesem Bildungstyp cf. Solèr 2003: 110).
} 
Switching ins Schweizerdeutsche in der Korrespondenz einer Teilnehmerin mit ihrem Freund, der erst Rätoromanisch lernt (19-21). In diesem letzten Fall muss zumindest teilweise von einem teacher code-switching gesprochen werden. Der deutsche Einschub kann einer Worterläuterung dienen (19) sowie der erleichterten Aufnahme eines Teils der Botschaft (in Beispiel 20 wird eine von zwei Fragen auf Schweizerdeutsch gestellt) oder sogar des Kerns der Botschaft (in Beispiel 21 wird die Frage, die zum Verfassen der SMS veranlasst, auf Schweizerdeutsch gestellt, während sich die Verwendung des Rätoromanischen auf den Rahmenteil - Begrüssung, Information zum örtlichen Befinden und Verabschiedung beschränkt $\left.{ }^{28}\right)$. In den beiden letzten Fällen (20/21) werden die Einschübe vermutlich auch der symbolischen Herstellung der Nähe dienen, ist doch das Schweizerdeutsche die Hauptsprache des Adressaten.

(18) Grazia fich, funcziunarà schon. "Gring abe u seckle" ;-) cs, Valerie (ID 683, SMS 11341, Vallader)

Danke vielmal, wird schon funktionieren. "Kopf tief und rennen";-) lg, Valerie

(19) Gea, tes sms vegnan adina dubel. Jau hai dafraid - verkeltet. E roger è malsaun [...] (ID 674, SMS 8163, Rumantsch Grischun)

Ja, deine sms kommen immer doppelt. Ich bin erkältet - [schwdt. Glossierung von erkältet]. Und roger ist krank [...]

(20) Ciao mes eddy, co èsi a militar? Ta legras sin tes anniversari? Pos ti dir a mai, quants che vegnan dumengia? Menü: pizza, dif. salatas, apero (chips etc.), biera, vin, dessert. Oder bringt öpert öppis mit? Ina bella saira ed in bitsch (ID 674, SMS 12039, Rumantsch Grischun)

Hallo mein eddy, wie ist es im militär? Freust dich auf deinen geburtstag? Kannst du mir sagen, wie viele am sonntag kommen? Menu: pizza, versch. salate, apero (chips etc.), bier, wein, dessert. Oder bringt jemand etwas mit? Schönen abend und kuss

(21) Ciao amur, nus essan grad en il tren enavos a fribourg. Ina dumonda: häsch luscht silvester bir cristallina z'verbringa- mit guido, mary etc. Guat essa und trinka, gmüatlich hocka. Bitsch da buna notg e dorma bain (ID 674, SMS 10555, Rumantsch Grischun)

Hallo mein liebster, wir sind gerade im zug zurück nach freiburg. Eine frage: hast $d u$ lust silvester bei cristallina zu verbringen- mit guido, mary etc. Gut essen und trinken, gemütlich zusammensitzen. Gutenachtkuss und schlaf gut

Während in Beispiel (21) der ganze Rahmenteil, der den Kern der Botschaft umgibt, rätoromanisch ist, so dass ein Code-Switching vom Rätoromanischen ins Deutsche stattfindet, enthält Beispiel (22) rätoromanische Einsprengsel im Rahmenteil (stest bain? "geht's dir gut?" und vessast vöglia? "hättest du lust"):

(22) Hoi stefi,stest bain?;)wie laufts so ir schuel?gell,du hesch au abem 18te ferie?wemer üs filicht wieder emol treffa?mier wärs egal ob no do in züri oder erst i de ferie,filicht denn au no mit de söci...was denksch?vessast vöglia?glg (ID 690, SMS 19260, Puter)

Diese SMS stammt von derjenigen rätoromanisch schreibenden Person (Nr. 690), die die meisten SMS verfasst hat (53), von diesen jedoch nur neun durchgängig auf Rätoromanisch. Als Muttersprache gibt diese Teilnehmerin nur das Schweizerdeutsche an, interessanterweise wechselt sie aber hier, in einer SMS, deren Empfängerin ebenfalls schweizerdeutscher

28 Angesichts dieses Verhältnisses haben wir diese SMS in unserer Statistik (cf. oben Tabelle 1) als schweizerdeutsch eingestuft. Bei der Übersicht über die Varietäten in den SMS (Tabelle 4) haben wir präzisiert, dass in einer schweizerdeutschen SMS dieser Person auch Rätoromanisch-Segmente vorkommen. 
Muttersprache zu sein scheint, ins Rätoromanische. Ist die Empfängerin ebenso zweisprachig wie die Verfasserin, so dass mit dem Code-Switching eine gemeinsame Gruppenzugehörigkeit markiert wird? Oder signalisiert die Verfasserin gegenüber ihrer (stärker deutschsprachigen) Korrespondentin ihre partielle Zugehörigkeit zur Gruppe der Rätoromanischsprachigen?

\section{Jugendsprachliche Merkmale}

Die SMS von zwei der drei Teenager und von elf der 13 Twens unseres Samples weisen, in unterschiedlicher Dichte, jugendsprachliche Merkmale auf.

Die folgenden Beispiele stammen von zwei Teenagern (weiblich) (23/24) und von einer 24jährigen Teilnehmerin (25):

(23) Hey norton!quei ei ina super ideja,aber sas tgei,jeu sun naven da venderdis studienwoche a tarifa e suenter in jamna maturarais!full schaiße.also venderdis en in jamna tochen dumengia sun jeu a casa...il rest da la fam ei aber cheu.peccato!ims ${ }^{29}$ biala sera julid (ID 1198, SMS 22051, Surselvisch)

Hey norton!das ist eine super idee, aber weisst du was,ich bin ab freitag studienwoche in tarifa und danach eine woche maturareise! full schaiße.also freitag in einer woche bis sonntag bin ich zu hause... der rest der fam ist aber hier.peccato!einen schönen abend julid

(24) Och man. Est sgür...? Uschglö scha at decidast oter venst aifach spontan, dhe reservo eir per te, provisorisch :) ma darius e florina nu paun eba gnir. Bütschuun adufg [= at d'he u fich gugent] (ID 667, SMS 7760, Puter)

Och mann. Bist du sicher...? Sonst wenn du dich anders entscheidest kommst du einfach spontan, habe auch für dich reserviert, provisoisch :) aber darius und florina können eben nicht kommen. Riesenkuuss hab dich so fest lieb

(25) Hei giönks. Fit? Sas sche ti vas? (ID 682, SMS 9557, Surselvisch)

Hei Junks. Alles klar? Weisst du, ob du gehst?

Die Beispiele enthalten markante Merkmale der deutschen Jugendsprache: die "attention getter" hey/hei (23/25) und (och) man (24) (vgl. Schlobinski/Kohl/Ludewigt 1993: 134) sowie den Vulgarismus full schaiße (24), wo voll Scheisse aufgrund der Anglisierung voll $\rightarrow$ full und der die phonetische Realisierung imitierenden Graphie ${ }^{30}$ schaiße verfremdet wird. Ausserdem treffen wir Merkmale der schweizerdeutschen Jugendsprache an: die Verstärkungspartikel $u$, die in die Grussformel adufg - für at d'he u fich gugent ("hab dich so fest lieb", 24) - eingeflochten ist, und die Verwendung von fit in der Frage nach dem allgemeinen Wohlergehen (25). Rätoromanisches Eis fit? folgt schweizerdeutschem Bisch fit?, das sich an ursprüngliches Bisch zwäg? ("Bist du wohlauf?") anlehnt und von diesem den Bezug auf das allgemeine - nicht nur körperliche - Wohlergehen übernommen hat (cf. Dürscheid/Spitzmüller 2006: 18). ${ }^{31}$ Ein weiteres jugendsprachliches Sprachkontaktphänomen zeigt sich in der Anrede giönks (25), wo englisches junks in rätoromanischer Graphie wiedergegeben wird. Neben spezifisch jugendsprachlichen Entlehnungen findet sich stilistisch weniger markiertes deutsches Wortgut, das jedoch gerade deshalb, weil es sich

\footnotetext{
${ }^{29}$ Eventuell ein Verschreiber für den unbestimmten Artikel ina (ina biala sera, "einen schönen Abend").

30 Zur Metagraphie in der rätoromanischen Jugendsprache cf. Derungs (2009: 80-85).

31 Im Rätoromanischen ersetzt Eis fit? (surselvisch)/Est fit? (engadinisch) als Frage nach dem allgemeinen Wohlergehen Eis en gamba?/Est in gamba? Der Ausdruck en/in gamba ist aus dem Italienischen (in gamba) entlehnt und war ursprünglich, aufgrund der Semantik in der Gebersprache wie englisches fit auf das körperliche Wohlergehen bezogen, hat dann aber - wohl ebenfalls aufgrund des Kontakts mit dem Schweizerdeutschen (zwäg) - die erweiterte Bedeutung 'wohlauf, in guter (körperlicher und seelischer) Verfassung' erhalten.
} 
kaum aufgrund lexikalischer Lücken motivieren lässt, einer Abgrenzung gegenüber der sprachpuristischen Haltung von Vertreterinnen und Vertretern der älteren Generationen dient: studienwoche und maturarais 'Maturareise, vor dem Abitur unternommene Klassenfahrt' (23) sind zwar Bezeichnungen, die sich aus dem Schulalltag aufdrängen, rätoromanische Äquivalente wären jedoch leicht verfügbar (jamna da studi 'Studienwoche', viadi da matura 'Maturareise'). Zu umgangssprachlich üblichem provisorisch (24) wurde das besonders nahe liegende rätoromanische Pendant provisori(c)amaing nicht bemüht, vielleicht auch wegen seiner Länge. Nicht vermieden wurden schliesslich der Germanismus aber sowie die ebenfalls aus dem Deutschen entlehnten Gesprächspartikel also (23), aifach 'einfach' und eba 'eben' (24), deren relativ hohe Frequenz im Gesprochenen gerade ein Merkmal der Jugendsprache ist (cf. Neuland 2008: 152, Dürscheid/Spitzmüller 2006: 21). Ein auffälliges Code-Switching stellt peccato! 'schade!' (23) dar, ist doch das Italienische heute nicht mehr eine Adstratsprache des Rätoromanischen. ${ }^{32}$ Dieses Einsprengsel erklärt sich vielleicht aus dem vorübergehenden Aufenthaltsort der surselvischen Verfasserin, die eine Mittelschule im Oberengadin besucht, wo das Italienische eine ziemlich starke Präsenz hat. Erwähnenswert ist auch ein innerrätoromanisches Code-Switching: die oberengadinische Form ideja 'Idee' in der auf Surselvisch verfassten Textnachricht (23). Ein nicht sprachkontaktbedingtes expressives Element ist die Augmentativbildung bütschuun 'Riesenkuss' (abgeleitet von bütsch 'Kuss' mit dem Suffix -un), wobei die expressive Komponente mit einem graphischen Mittel - durch Vokalverdoppelung im Augmentativsuffix - zusätzlich verstärkt wird (24). Bütschuun 'Riesenkuss' ist auch eine Parodie des mit gelängten Vokalen ausgesprochenen Diminutivs bütschins 'Küsschen' (Pl.), mit dem sich die Fernsehmoderatorin Tonia Maria Zindel nach der bis Ende 2009 ausgestrahlten rätoromanischen Gutenachtgeschichte jeweils vom Publikum verabschiedete.

Abschliessend sei auf Ökonomieverfahren auf der syntaktischen Ebene hingewiesen, die vermutlich durch das Kommunikationsmedium Mobiltelefon begünstigt werden. In Beispiel (23) werden in jeu sun naven da venderdis studienwoche ("ich bin ab freitag studienwoche") Präposition und Artikel ausgelassen (ella/en ina studienwoche, "in der/in einer Studienwoche"). Die "vervollständigte" Konstruktion offenbart eine frappante Lehnsyntax. Für ich bin in der/einer Studienwoche wäre rätoromanisches - nicht so viele Zeichen beanspruchendes - jeu hai l'/in'jamna da studi ("ich habe die/eine Studienwoche") angebracht. In Beispiel (25) wird das Prädikatsadjektiv fit im Fragesatz unter Auslassung der Kopula eis 'bist (du)' verwendet, womit die Mitteilung einen sehr informellen Charakter erhält. Jugendsprachlich geprägt sind auch die SMS einer 27-jährigen (26/27) und einer 28jährigen Teilnehmerin (28):

(26) Hallöchen:-)!stau u cool a saas-fee:-),schizun vegni delectai cun cant celestial giu da breil;-).e vus,giu ina biala fin d'jamna?ina sera da giugs tuna super!setelefoneinsa!zera hai eba cuors da far cun arschella,aber damaun dun in tel.bunet:-) (ID 832, SMS 11176, Surselvisch)

Hallöchen:-)!echt cool gewesen in saas-fee:-),sind sogar mit himmlischem Gesang aus Brigels erfreut worden;-).und ihr,ein schönes wochenende gehabt?ein spielabend tönt super!wir rufen uns an!heute abend habe ich eben tonmodellierkurs,aber morgen rufe ich an.küsschen:-)

(27) Bellezia,sc'ina flitzra culla jacca nova,e ferdabrraglias!ed il fadil ei staus jenes geschmeichelt ;-).biala fin d'jamna è a vus 2 cars!bunets (ID 832, SMS 11181, Surselvisch)

\footnotetext{
32 Zum historischen Sprachkontakt mit dem Italienischen cf. Diekmann 1982, Kristol 1985, Popovici 2006
} sowie Grünert 2003, 2006 und 2009. 
Wunderschön [war sie],wie eine flitzerin mit der neuen jacke, und eisskalt [war es]!und fadil war jenes geschmeichelt ;-).auch euch 2 lieben schönes wochenende!küsschen

(28) Her d'eira easy. Temp pero per novas chanzuns. La russa cha tü hast manà nu vaivna. Lultim d'eran be amo lau, bernadette ed eu vain fat sul na geila impro-jam. I dà darcheu ün doodle per la prosma. Va per medis schi nu vegn meglder. Buna not (ID 837, SMS 11231, Vallader)

Gestern war's easy. Aber zeit für neue songs. Der russische, den du mitgebracht hast, hatten sie noch nicht. Am schluss waren nur noch lau, bernadette und ich haben echt 'ne geile Impro-jam gemacht. Es gibt wieder ein doodle für die nächste. Geh medis holen, wenn's nicht bessert. Gute nacht

In dieser zweiten Reihe von Beispielen zur Veranschaulichung jugendsprachlicher Elemente (26-28) ist einerseits der kreative Umgang mit der Sprache bei einer der beiden Teilnehmerinnen (26/27) bemerkenswert, andererseits ein Aspekt der morphologischen Assimilation von Entlehnungen, der sich beim Substantiv flitzra 'Flitzerin, Raserin' (27) und beim Adjektiv geila 'geil' f. (28) zeigt. Die Kreativität äussert sich auf unterschiedlichen Ebenen: in einer Stilmischung, in der auf Analogie basierenden, bisher nicht üblichen reflexiven Verwendung eines Verbs sowie im Einschub einer onomatopoetischen Lautverbindung im Innern eines Wortes:

- Eine Stilmischung, genauer eine die Jugendsprache charakterisierende Bricolage oder "Stil-Bastelei" (cf. Neuland 2008: 71f., 149-151), liegt in Beispiel (26) vor, wo der stilistisch gehobene Passus zum Genuss geistlichen Gesangs (delectai cun cant celestial, "mit himmlischem Gesang erfreut") mit dem Bericht zu einem lockeren Urlaub (stau u cool a saas-fee, "echt cool gewesen in saas-fee") kontrastiert. Die Referenz des "himmlischen Gesangs", der "von Brigels herab" erklingt, wird ein in Brigels in der Surselva stattfindendes geistliches Chorkonzert sein, in das die Korrespondentinnen eventuell persönlich involviert sind und von dem im entfernten Urlaubsort eine Aufnahme gehört wird.

- Die reflexive Adhortativform setelefoneinsa! 'rufen wir uns an!'/' wir rufen uns an!' in Beispiel (26) ist eine spontane Innovation, die auf sevesinsa 'wir sehen uns!' basiert, zu welchem - in der E-Mail-Kommunikation - analog bereits sescrivinsa 'wir schreiben uns!' aufgekommen ist. Das im Surselvischen für alle Personen generalisierte und ans Vollverb gebundene (d.h. in den zusammengesetzten Zeiten nicht vor das konjugierte Auxiliar gesetzte ${ }^{33}$ ) Reflexivum se- ist nur eingeschränkt - bei Verben mit einem direkten Personenobjekt, tendenziell bei Wahrnehmungsverben - für den Ausdruck der Reziprozität verfügbar: seveser 'sich (gegenseitig) sehen, sich/einander wiedersehen', s'observar 'sich (gegenseitig) beobachten, einander beobachten', jedoch nicht *s'enconuscher 'sich (gegenseitig) kennen, einander kennen'. Alternativ kommt die Formel in(a) (a) l'auter/l'autra 'einander' zur Anwendung, die bei Verben mit nur indirektem Personenobjekt der einzige nach der Gebrauchsnorm verfügbare Ausdruck ist: rispunder in(a) a l'auter/l'autra 'sich/einander antworten', scriver in(a) a l'auter/l'autra 'sich/einander schreiben' (cf. Strebel 2005: 84-88 und Appendix XII). Bei dem vorliegenden Verb telefonar, das nur ein indirektes Personenobjekt nach sich ziehen kann, wäre tatsächlich telefoneinsa in(a) a l'auter/l'autra! für 'wir rufen uns an!' üblich.

33 Z.B. surselvisch nus essen sevesi(das) 'wir haben uns gesehen' vs. engadinisch nus ans avain(s) vis(sas), fr. nous nous sommes vu(e)s, it. ci siamo visti/e. 
- In Beispiel (27) ist ins Wort ferdaglia 'Kälte' die onomatopoetische Lautverbindung brr eingefügt (ferdabrraglias), womit der beschriebene Sachverhalt mit einem Kommentar versehen wird. Der Einschub ins Wortinnere unterstreicht das Bedürfnis, den Sachverhalt gleichzeitig zu beschreiben und emotional zu kommentieren.

Die morphologische Assimilierung, die sich bei femininen Formen von Entlehnungen manifestiert - hier bei der Bildung flitzra 'Flitzerin, Raserin' (27), wo das deutsche Genussuffix -in durch rätoromanisches - $a$ ersetzt wird, und beim Adjektiv geila 'geil' f. (28) -, wirken aufgrund ihrer Verbindung eines deutschen lexikalischen Morphems und eines rätoromanischen grammatischen Morphems, das eine eigene Silbe bildet, provokativer als isolierte lexikalische Morpheme (z.B. mask. flitzer, geil, ohne grammatische Morpheme) oder Entlehnungen, die lediglich ein Plural-s haben (welches zwei wichtige Gebersprachen, das Deutsche und das Englische, ebenfalls kennen). Die Hybridität scheint bei den zitierten Feminina stärker wahrgenommen zu werden.

Ausser diesen markanten Merkmalen enthält die zweite Reihe von Beispielen (26-28) die bereits oben erwähnten Charakteristiken. Als Sprachkontaktphänomene seien einerseits jugendsprachliche Entlehnungen genannt - die affektiv modifizierte Begrüssung Hallöchen (26), die Verstärkungspartikel $u$ (26) und jenes (27) sowie die Adjektive cool (26) und easy (28) -, andererseits weniger markierte Entlehnungen, die ebenfalls zu einer Distanzierung von der puristischen Schriftnorm beitragen, darunter graphisch und morphologisch angepasstes jacca (von dt. Jacke) anstelle von giacca, geschmeichelt, zu dem der wenig verbreitete Gallizismus flattau eine Alternative böte (27), die nicht ersetzbaren Internationalismen improjam und doodle sowie die aus dem Schweizerdeutschen übernommene Kurzform medis 'Medikamente' (28). Expressive Elemente aus eigensprachlichem Material sind der Diminutiv bunet 'Küsschen' (abgeleitet von buna 'Kuss', 26/27) und das als Verstärkungslexem verwendete Adjektiv/Adverb sul 'unheimlich' (28). Ein sehr informeller Sprachgebrauch wird mit der Verkürzung des unbestimmten Artikels üna zu na (28) signalisiert. Die Stellung dieses Artikels nach dem Verstärkungslexem sul verrät schliesslich deutsche Lehnsyntax: sul na geila impro-jam ("echt 'ne geile Impro-jam") statt der rätoromanischen Wortfolge (ü)na sul geila impro-jam.

\section{$7 \quad$ Ellipsen}

In den rätoromanischen SMS fallen zahlreiche Ellipsen auf, nicht nur vor dem Hintergrund der jeweiligen durch die Schule vermittelten regionalen Schriftnorm, sondern teilweise auch vor dem Hintergrund der sprechsprachlichen Norm. Die unten stehenden Beispiele sollen einen Überblick über die ausgelassenen Kategorien geben, die jeweils in Zitat und Übersetzung in eckigen Klammern ergänzt sind.

In den beiden ersten Beispielen fehlen Artikel (29) und Präpositionen in Verbindung mit dem Artikel (30). Ausgelassenes präsentatives esser 'sein' (29) könnte auch in der Sprechsprache fehlen.

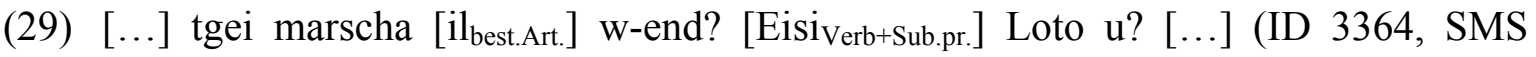
14013, Surselvisch)

[...] was läuft [am] w-end? [Gibt's ein] Lotto nicht wahr? [...]

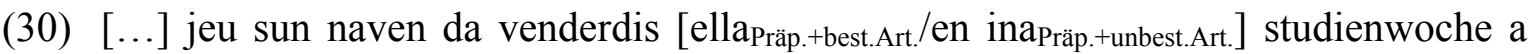
tarifa e suenter in jamna [sillapräp.+best.Art.] maturarais! [...] (ID 1198, SMS 22051, Surselvisch)

[...] ich bin ab freitag [in der/in einer] studienwoche in tarifa und danach eine woche [auf der] maturareise! [...] 
Während Artikel und Präpositionen sporadisch ausgelassen werden, ist die Ellipse des proklitischen Subjektpronomens, die in den folgenden Beispielen veranschaulicht wird, ein sehr häufiges, für die rätoromanische SMS-Kommunikation charakteristisches Phänomen.

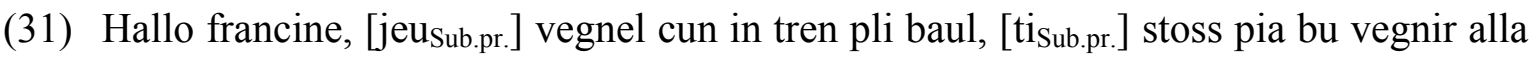
staziun. (ID 899, SMS 22377, Surselvisch)

Hallo francine, [ich] komme mit einem zug früher, [du] musst also nicht an den bahnhof kommen. [...]

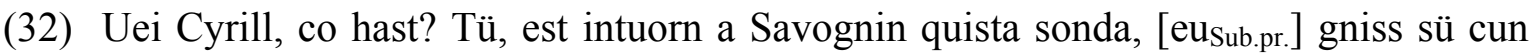

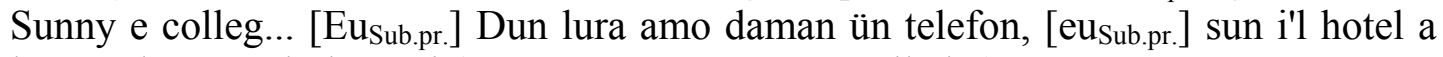
lavurar hoz... Salüds Jessi (ID 1196, SMS 22219, Vallader)

Hallo Cyrill, wie geht's dir? Du, bist du anzutreffen in Savognin ${ }^{34}$ diesen samstag, [ich] käme hinauf mit Sunny und Kollege... [Ich] rufe dann morgen noch an, [ich] bin heute im Hotel am Arbeiten ... Grüsse Jessi

Bei der Auslassung des Subjektpronomens handelt es sich nicht um eine Neuerung, sondern um einen bereits in der Literatur des 16. und 17. Jahrhundert im Engadin - allerdings nur für die 1., 3. und 6. Person - bezeugten Gebrauch (cf. Linder 1987: 22f.). Im 19. Jahrhundert ist dieser auch für die 2. und 4. Person belegt. Bei Giovannes Mathis, einem Engadiner Autor des 19. Jahrhunderts, bezeichnet Linder (1987: 23) die Ellipse des Subjektpronomens als Marotte, "die von heutigen Sprechern nicht mehr akzeptiert wird". Im Zuge des antiitalienischen Purismus im Engadin in den 1910er bis 1930er Jahren wurde diese Eigenheit der Schriftsprache als Italianismus kritisiert und verschwand darauf weitgehend aus der Literatur (cf. Linder 1987: 24f., 31). Linder sieht in der Ellipse des Subjektpronomens auch ein Phänomen, "das den Briefstil des 19. Jahrhunderts charakterisiert, ein stilistisches Phänomen, das wir auch im Deutschen finden: „, Sind gut angekommen“ statt „,Wir sind... “, „Habe soeben mit XY telefoniert" statt „Ich habe... “ etc." (Linder 1987: 21). Während allerdings die Auslassung des Subjektpronomens der 1. und 4. Person im Briefstil ihre Begründung in der Zurücknahme der Schreibenden hatte, die sich aus Höflichkeit den Adressaten gegenüber nicht nennen wollten (Reh 2003: 214), ist die Ellipse des Subjektpronomens im SMSSprachgebrauch, zumindest anfänglich, ein Verfahren, das dem Einsparen von Platz und von Zeitaufwand dient. ${ }^{35}$ Diese Motivation legt eher eine Verbindung zum Telegrammstil nahe (cf. "Ankomme Freitag 17 Uhr" für "Ich komme am Freitag um 17 Uhr an", wo mit dem Auslassen von Wörtern Kosten gespart wurden). Bezüglich des in der deutschen SMSKommunikation häufigen Wegfalls des nicht emphatisch hervorgehobenen Subjektpronomens im Vorfeld (_ Bin gleich wieder da) bemerkt Dürscheid (2002: 15), dass die Konstruktion an sich nicht neu sei, dass die Neuigkeit lediglich in der Häufigkeit des Auftretens liege. Wie im Deutschen lässt sich auch im Rätoromanischen die erneute Zunahme eines bereits zu einer früheren Zeit verbreiteten Phänomens feststellen. Die Verhältnisse in unserem SMS-Korpus nähern sich also den in der engadinischen Schriftsprache des 19. und des Beginns des 20. Jahrhunderts beobachteten Verhältnissen an und heben sich somit deutlich von der neueren schriftsprachlichen Praxis ab, die Linder (1987: 21) zu folgendem Schluss kommen liess: "Die Auslassung des Subjektpronomens, da wo es heute im Bündnerromanischen in proklitischer Stellung zu stehen käme, ist ausgesprochen selten".

\footnotetext{
34 Rätoromanisches est intuorn (a Savognin) lässt sich besser mit schweizerdeutschem bisch ume (z Savognin) wiedergeben, von dem es eine Lehnübersetzung darstellt. Schweizerdeutsches ume si (wörtl. herum sein) bedeutet 'präsent sein', 'anzutreffen sein'.

35 Dass dies inzwischen nur noch teilweise zutrifft, hält Dürscheid (2003: 235) fest: "Es sind also nicht mehr nur Ökonomieanforderungen, die hier eine Rolle spielen. Vielmehr geht es auch darum, den an die Kommunikationsform herangetragenen Erwartungen gerecht zu werden".
} 


\begin{tabular}{|c|c|c|c|c|c|c|c|}
\hline & & $\begin{array}{l}\text { Sursel- } \\
\text { visch }\end{array}$ & $\begin{array}{l}\text { Surmei- } \\
\text { risch }\end{array}$ & Puter & $\begin{array}{l}\text { Valla- } \\
\text { der }\end{array}$ & $\begin{array}{l}\text { Rumantsch } \\
\text { Grischun }\end{array}$ & Total \\
\hline \multirow{2}{*}{ 1. Person } & gesetzt & $18 \quad 21 \%$ & 1 & 10 & 3 & 19 & $35 \%$ \\
\hline & ausgel. & $6736 \quad 79 \%$ & 3 & 9 & 15 & 2 & $96 \quad 65 \%$ \\
\hline \multirow{2}{*}{ 2. Person } & gesetzt & 11 & & & 4 & 3 & 18 \\
\hline & ausgel. & 4 & & 3 & & & 7 \\
\hline \multirow{2}{*}{ 4. Person } & gesetzt & 9 & & 4 & 2 & 2 & 17 \\
\hline & ausgel. & 15 & 2 & & & & 17 \\
\hline \multirow{2}{*}{ 5. Person } & gesetzt & 4 & & & & & 4 \\
\hline & ausgel. & 1 & & & & & 1 \\
\hline
\end{tabular}

Tabelle 7: Proklitisches Subjektpronomen

In unserem kleinen Korpus sind besonders die Daten zur gut belegten 1. Person aussagekräftig, bei der das nicht emphatisch hervorgehobene Subjektpronomen in zwei Dritteln aller Fälle ausgelassen wird. Auffällig ist die gegenläufige Tendenz in den auf Rumantsch Grischun verfassten SMS. 17 der 19 Setzungen des Pronomens sowie die beiden Ellipsen gehen auf das Konto der Hauptverfasserin (Nr. 674) von SMS auf Rumantsch Grischun. Die beharrliche Verwendung des Pronomens kann man damit erklären, dass sich diese Verfasserin bemüht, gegenüber ihrem Freund, der erst Rätoromanisch lernt, eine normkonforme Varietät zu verwenden. Die beiden Ellipsen befinden sich bezeichnenderweise in zwei Nachrichten, welche diese Verfasserin an eine andere Person geschickt hat.

In der Proklise kann im Engadinischen ausser dem Subjektpronomen auch das Objektpronomen wegfallen. Die beiden Ellipsen können zusammen vorkommen, wie im folgenden Beispiel:

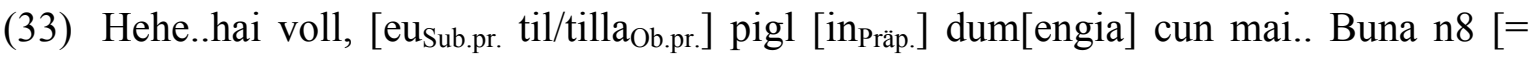
not] (ID 3331, SMS 10401, Vallader)

Hehe..ja voll, [ich] nehme [ihn/sie] [am] so[nntag] mit ${ }^{37}$.. Gute n8 [= nacht]

In den surselvischen SMS ist die Ellipse der Auxiliarverben des zusammengesetzten Perfekts und der Kopula belegt, stets in Verbindung mit der Ellipse des Subjektpronomens:

(34) Hallöchen:-)![igl $\left.\right|_{\text {Sub.pr. }}$ ei $i_{\text {Aux. }}$. stau u cool a saas-fee:-),[nus Sub.pr. $_{\text {essan }}$ Aux. $_{\text {S }}$ schizun vegni delectai cun cant celestial giu da breil;-).e vus,[veis Aux. [vus $_{\text {Sub.pr.] }}{ }^{38}$ giu ina biala fin d'jamna? [...] (ID 832, SMS 11176, Surselvisch)

Hallöchen:-)![es ist] echt cool gewesen in saas-fee:-),[wir sind] sogar mit himmlischem Gesang aus Brigels erfreut worden;-).und ihr,[habt ihr] ein schönes wochenende gehabt? [...]

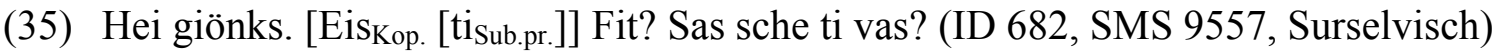
Hei Junks. [Bist du] wohlauf? Weisst du, ob du gehst?

Während im Deutschen nur das enklitische Subjektpronomen der 2. Person ausgelassen zu werden scheint (cf. oben Fussnote 15), kennt das Rätoromanische die Ellipse des enklitischen Subjektpronomens der 1., 2., 4. und 5. Person. In diesem Zusammenhang sei auf die beachtliche Frequenz der enklitischen Position im Rätoromanischen hingewiesen, die daraus resultiert, dass das Rätoromanische, wie das Deutsche, eine Verb-Zweit-Sprache ist (cf.

\footnotetext{
${ }^{36}$ Hier sind zwei Verwendungen aus dem einzigen sutselvischen Beleg (cf. oben Beispiel 4) eingeschlossen.

37 Wörtlich: cun mai, "mit mir".

${ }^{38} \mathrm{Da}$ in der Umgangssprache hier sowie in Beispiel (35) das enklitische Subjektpronomen nicht verwendet würde, setzen wir es in eine zusätzliche Klammer.
} 
Kaiser 2003: 312). Die Ellipse in der Enklise kann allerdings im Rätoromanischen höchstens teilweise als charakteristisch für die SMS-Kommunikation gelten. In der 2. und 5. Person ist nämlich die Auslassung des enklitischen Subjektpronomens besonders häufig (cf. Ganzoni 1977: 64, Spescha 1989: 334, 563 und Kaiser/Hack 2010: 87-89), wobei sich eine Abstufung zwischen der regelmässigen Auslassung in der engadinischen Sprechsprache (cf. Linder 1987: 53), und der sporadischen Setzung im Surselvischen - mit einer Differenzierung zwischen seltenerem Gebrauch in der Sprechsprache und häufigerem Gebrauch in der Schriftsprache beobachten lässt. Die sich in unserem Korpus ergebenden Verhältnisse bei der 2. und 5. Person (dazu die Beispiele 36/37) sind also keineswegs spezifisch für die SMSKommunikation, sondern veranschaulichen, besonders beim Surselvischen, den konzeptionell mündlichen Charakter dieses Schreibens. Auffällig ist in unserem Korpus auch der relativ hohe Anteil von Auslassungen bei der 1. Person (dazu die Beispiele 38/39), bei der die schriftsprachliche Norm die Setzung des Pronomens verlangt, während in der Sprechsprache die Ellipse vorkommt, jedoch deutlich weniger häufig als bei der 2. Person. Unterschiedliche Tendenzen in der 1. und 2. Person weisen auch Kaiser und Hack (2010: 88) in ihrer Untersuchung zum umgangssprachlichen Gebrauch nach. Eine gute Hälfte ihrer Befragten, die in einem Fragebogen mit Satzbeispielen mit und ohne enklitischem Subjektpronomen konfrontiert wurden, gab in Bezug auf die 1. Person an, dass sie die Variante ohne Subjektpronomen verwenden würde; bei der 2. Person waren es dagegen vier Fünftel, die der Variante ohne Subjektpronomen den Vorzug gaben.

\begin{tabular}{|c|c|c|c|c|c|c|c|}
\hline & & $\begin{array}{l}\text { Sursel- } \\
\text { visch }\end{array}$ & $\begin{array}{l}\text { Surmei- } \\
\text { risch }\end{array}$ & Puter & $\begin{array}{c}\text { Valla- } \\
\text { der }\end{array}$ & $\begin{array}{c}\text { Rumantsch } \\
\text { Grischun }\end{array}$ & Total \\
\hline \multirow{2}{*}{ 1. Person } & gesetzt & 5 & 1 & 10 & 3 & 19 & 19 \\
\hline & ausgel. & 13 & 3 & 9 & 15 & 2 & 17 \\
\hline \multirow{2}{*}{ 2. Person } & gesetzt & 4 & & 2 & & 2 & $10 \%$ \\
\hline & ausgel. & 27 & 1 & 19 & 11 & 12 & $90 \%$ \\
\hline \multirow{2}{*}{ 4. Person } & gesetzt & 2 & & 1 & 2 & & 5 \\
\hline & ausgel. & 8 & & 2 & 2 & & 12 \\
\hline \multirow{2}{*}{ 5. Person } & gesetzt & & & & & & \\
\hline & ausgel. & 8 & & 1 & 2 & & 11 \\
\hline
\end{tabular}

Tabelle 8: Enklitisches Subjektpronomen

(36) Tgau tü, eu dun lura ün tel. Pel mumaint na sun[a asub.pr.] propcha fit. Ma ün dus

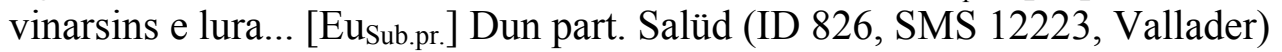
Ciao du, ich rufe dann an. Im moment bin [ich] nicht gerade fit. Aber ein zwei schnäpschen und dann... [Ich] gebe bescheid. Gruss

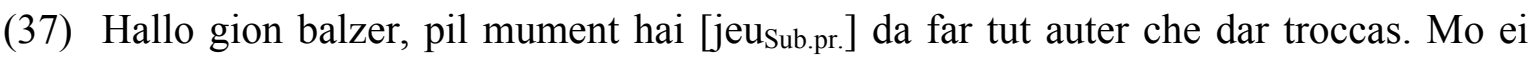
dat lu schon puspei temps persuenter. Oz sun [jeu Sub.pr.] $_{\text {stada a berna, [jeu }}$ Sub.pr.] sun el tren viers casa. Biala sera e cs lucrezia (ID 899, SMS 16611, Surselvisch)

Hallo gion balzer, im moment habe [ich] alles andere zu tun als tarock zu spielen. Aber es gibt dann schon wieder zeit dafür. Heue bin [ich] in bern gewesen, [ich] bin im zug nach hause. Schönen abend und lg lucrezia

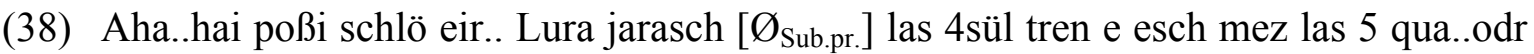

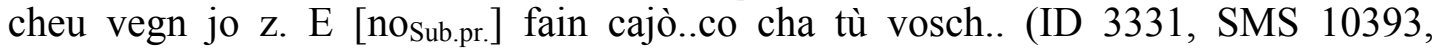
Vallader)

Aha..ja kann ich sonst auch.. Dann gehst [du] um 4auf den zug und bist um halb 5 hier..oder ich komme nach z[ürich] hinunter. Und [wir] machen [es] dort unten..wie du willst.. 


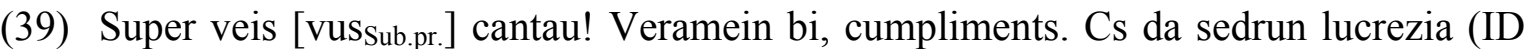
899, SMS 20489, Surselvisch)

Super habt ihr gesungen! Wirklich schön, komplimente. Lg aus sedrun lucrezia

In Beispiel (36) zum Vallader erscheint das ergänzte enklitische Pronomen der 1. Person in einer phonetisch reduzierten Form $-a[\mathrm{e}]$, und hebt sich somit vom proklitischen Pronomen eu $[\varepsilon(u)]$ ab. In Beispiel (37) zum Surselvischen, wo ebenfalls beide Positionen vorkommen, ist kein entsprechender Unterschied sichtbar, weil im Surselvischen das phonetisch reduzierte enklitische Subjektpronomen der 1. Person, $[\mathrm{u}]$ bzw. nach Vokal [u], in der Standardorthographie - an die sich auch alle Teilnehmenden der Untersuchung gehalten haben - nicht notiert wird. Neben schriftsprachlichem sun(del) jeu 'bin ich' und hai(el) jeu 'habe ich' sind z.B. in der Presse ${ }^{39}$ folgende konzeptionell mündlichen Varianten bezeugt: sund'jeu, die Formen mit dem Morphem der 1. Person - $\mathrm{el}^{40}$ und der Ellipse des enklitischen Pronomens (sundel, haiel) sowie die Formen mit anlautendem $v$ - zu haver 'haben' (vai jeu, vaiel [jeu] $)^{41}$. Die phonetischen Realisierungen sind ['sun(d)jeu] (cf. DRG 5: 712),

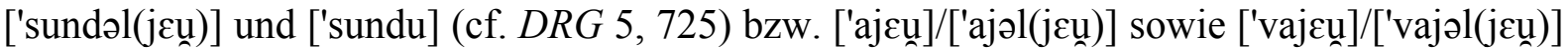
und [vau]. Neben den sprechsprachlich markierten Formen, die bereits Eingang in gewisse Register der Schriftsprache gefunden haben, wären also, als an die Aussprache angelehnte Graphien, auch sundu und vau denkbar. Diese kommen jedoch in unserem Korpus nicht vor. Die synthetische, das enklitische Pronomen einschliessende Form vau würde nicht einmal mehr Zeichen beanspruchen als die in Beispiel (37) verwendete Verbalform hai, nach der das Subjektpronomen ausgelassen ist. Die Tendenz zur Ellipse des Subjektpronomens (bei welcher die gewohnte Graphie der Verbalformen belassen wird) scheint stärker zu sein als die Neigung zur phonetischen Wiedergabe von ausdrücklich Sprechsprachlichem.

\section{Abkürzungen}

Abschliessend geben wir eine Übersicht über die Abkürzungen, die im rätoromanischen Teil des SMS-Korpus auftreten. Aus den vorliegenden Daten kann geschlossen werden, dass das Verkürzen stark in Anlehnung ans Deutsche praktiziert wird, dass also Abkürzungen, die sich zum Teil von Region zu Region unterscheiden, gerade deshalb leicht dekodierbar sind, weil in denselben pragmatischen Kontexten bzw. für dieselben Inhalte wie im Deutschen abgekürzt wird. Eine prominente Stellung haben verkürzte Verabschiedungsfloskeln, die Liebes- und Freundschaftsbezeugungen enthalten können oder auch alleine aus solchen bestehen können. Zur Hauptsache handelt es sich dabei um Akronyme, die in Anlehnung an deutsche Akronyme entstanden sind, wie $c s=$ cars salids/chars salüds, das deutschem $l g=$ liebe grüsse entspricht. Das $c$ in $c s$ wird zwar bisweilen auch als cordials 'herzliche' (pl.) glossiert, in der vor allem informellen SMS-Kommunikation ist es wohl aber häufiger als cars/chars 'liebe' (pl.) zu verstehen. Die Abkürzung $c s$ ist die einzige wirklich etablierte: Sie wird von 14 Teilnehmenden, d.h. fast von der Hälfte aller rätoromanisch Schreibenden, in insgesamt 44 Botschaften verwendet. Die anderen Abkürzungen sind jeweils nur bei einer Person belegt, wenn man von buna $n 8$ = buna not $(g)$ 'gute Nacht' absieht, das zweimal vorkommt. Auch diese Rebusschreibung, die darin besteht, dass ein Zahlzeichen die dem Zahlwort entsprechende Buchstabenfolge ersetzt, hat ein deutsches Vorbild, gute n8: Die Acht - im Surselvischen otg [ot6] und im Vallader ot - deckt in der Schreibung not $(g)$ die auf den Anfangsbuchstaben $n$ folgenden Buchstaben ab, ebenso wie dies im deutschen Nacht der Fall

\footnotetext{
39 Im Online-Archiv der rätoromanischen Tageszeitung La Quotidiana, wo alle Ausgaben ab Juni 1997 verfügbar sind (www.suedostschweiz.ch/archiv, Stand 19.9.2011).

${ }^{40}$ Während dieses Morphem bei den regelmässigen Verben im Indikativ Präsens und Imperfekt obligatorisch ist, bestehen bei verschiedenen unregelmässigen Verben im Indikativ Präsens Varianten mit und ohne -el.

41 Anlautendes $v$ - in (jeu) vai '(ich) habe' erklärt sich aus Analogie zu den Pluralformen (nus) vein 'wir haben' und (vus) veis 'ihr habt' oder aus $u$ von EGO > eu (cf. Decurtins 1958: 151).
} 
ist (im Puter, wo die beiden Wörter och [otc] und not lauten, wäre diese Graphie nicht möglich).

Die Akronyme adg, adfg und admmmfg lassen sich neben die (schweizer)deutschen Akronyme $h d g=h a$ di gern, $h d l=h a$ di lieb/hab dich lieb sowie Varianten mit den Verstärkungslexemen fest und mega stellen. Rätoromanisches (Puter) $a t_{\text {Ob.pr. }} d^{\prime} h e_{\text {Verb }}$ gugent $_{\mathrm{Adv}}$. kommt schweizerdeutschem $h a_{\mathrm{Verb}} d i_{\mathrm{Ob} . p r .}$ gern $_{\mathrm{Adv}}$. besonders nahe (gegenüber dem Ausdruck hab dich lieb), entspricht doch die semantische Extension von (avair) gugent weitgehend derjenigen von gern $(h a)$. In beiden Fällen wird zudem das Subjektpronomen weggelassen, so dass ein dreigliedriger Basisausdruck entsteht, der sich mit (multiplizierbaren) Verstärkungslexemen kombinieren lässt. Als Verstärkungslexem in $a d f g$

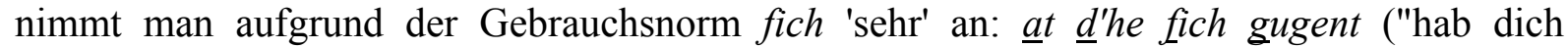
sehr/fest gern"). Im Fragebogen liefert allerdings die Teilnehmerin eine Glossierung der Variante $a d m m m f g$, in der sie $f$ als ferm 'stark' erklärt, was einer zusätzlichen Intensivierung gleichkommt.

\begin{tabular}{|l|l|l|}
\hline Rätorom. & $\begin{array}{l}\text { Anlehnung } \\
\text { an Schw(dt.) }\end{array}$ & Glossierung und Belege im Korpus \\
\hline cs & $l g$ & $\begin{array}{l}\text { surselv. cars/cordials salids, engad. chars/cordials salüds, rum. } \\
\text { grisch. chars/cordials salids ("liebe/herzliche Grüsse") [von 14 } \\
\text { Teilnehmenden insgesamt 44 Mal verwendet] }\end{array}$ \\
\hline cseb & $\lg u k$ & $\begin{array}{l}\text { rum. grisch. chars salids e bitschs, surselv. cars salids e bunas } \\
\text { ("liebe Grüsse und Küsse") (ID 674, SMS 9404 / 10717 / 12299 } \\
\text { / 12594 / 18338) }\end{array}$ \\
\hline cs b & & $\begin{array}{l}\text { rum. grisch. chars salids, bitsch ("liebe Grüsse, Küsse") (ID } \\
\text { 697, SMS 8647) }\end{array}$ \\
\hline sal & & engad. salüds ("Grüsse") (ID 837, SMS 11718 / 12509) \\
\hline adg & $h d g, h d l$ & puter at d'he gugent ("hab dich gern") (ID 690, SMS 8286) \\
\hline adfg & $h d f g, h d f l$ & $\begin{array}{l}\text { puter at d'he fich gugent ("hab dich fest gern") (ID 690, SMS } \\
\text { 8287) }\end{array}$ \\
\hline admmmfg & $\begin{array}{l}\text { hdmmmfg, } \\
\text { hdmmmfl }\end{array}$ & $\begin{array}{l}\text { puter at d'he mega mega mega ferm gugent ("hab dich mega } \\
\text { mega mega fest gern") (ID 690, nur in einer Bemerkung im } \\
\text { Fragebogen) }\end{array}$ \\
\hline ct & $l d$ & surselv. carezel tei ("liebe dich") (ID 3364, SMS 14013) \\
\hline bffenl & & $\begin{array}{l}\text { eventuell surselv. betschel fetg ferm ("küss dich ganz fest"), } \\
\text { weitere Dekodierung völlig unklar (ID 3364, SMS 14013) }\end{array}$ \\
\hline buna $n 8$ & gute $n 8$ & $\begin{array}{l}\text { surselv. buna notg / vallader buna not ("gute Nacht") (ID 680, } \\
\text { SMS 13809; ID 3331, SMS 10401) }\end{array}$ \\
\hline
\end{tabular}

Tabelle 9: Abkürzungen von Verabschiedungs- und Liebesbezeugungsformeln

Eine weitere semantische Gruppe bilden abgekürzte Zeitangaben. Zwei Abkürzungen von Wochentagen sind belegt, me = mesjamna 'Mittwoch' (ID 832, SMS 11177) und dum = dumengia 'Sonntag' (ID 721, SMS 9048; ID 3331, SMS 10401), in partieller Anlehnung an den in anderen Sprachen verbreiteten Usus, die Wochentage mit den ersten Buchstaben und ohne Punkt abzukürzen (bei dum = dumengia mag die Verwendung der ersten drei Buchstaben erstaunen, wäre doch mit den ersten beiden, $d u$, bereits die erste Silbe repräsentiert). Die nur bei einem Verfasser auftretenden Akronyme für die Tageshälften, am

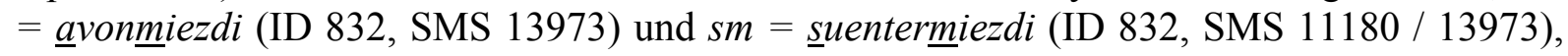
folgen dem deutschen Vorbild $V m=\underline{\text { Vormittag und }} \overline{N m}=\underline{\text { Nachmittag. Schliesslich findet }}$ sich die entlehnte Abkürzung w-end (cf. das obige Beispiel 5). 
Unter den übrigen Abkürzungen registrieren wir zwei spontane rätoromanische Abkürzungen mit Punkt, engr. = 'Danke' (ID 977, SMS 14238) und dif. = different(a)s 'verschiedene' (pl., ID 674, SMS 12039), die aus dem Deutschen entlehnte Abkürzung evtl. (ID 977, SMS 14230), die im rätoromanischen Text als eventualmein zu lesen ist, sowie die internationale Abkürzung tel., die je nach Kontext für das Substantiv telefon - mit den Bedeutungen 'Anruf' (ID 826, SMS 12223; ID 832, SMS 11176) und 'Telefongerät' (ID 3222, SMS 12429) - oder für das Verb steht: telefonar 'telefonieren' (ID 680, 13809), telefoneschel '(ich) telefoniere' (ID 680, SMS 8050; ID 3222, 17967).

\section{$9 \quad$ Schluss und Ausblick}

Die zum Rätoromanischen vorliegenden Daten haben uns erlaubt, varietätenlinguistische und sprachkontaktbezogene Fragestellungen zu skizzieren. Die Abweichungen von der regionalen Schriftnorm in den rätoromanischen SMS ergeben sich durch die Verwendung von Mundartlichem, durch die Wiedergabe phonetischer und morphosyntaktischer Merkmale der regionalen Sprechsprache sowie aufgrund der für die Sprechsprache allgemein charakteristischen lexikalischen und syntaktischen Germanismen, die ihrerseits zu einem grossen Teil regionalspezifisch ausgeprägt sind. Allgemeine Tendenzen mit regionalspezifischen Ausprägungen lassen sich auch in jugendsprachlichen Merkmalen, bei den für die computergesteuerte Kommunikation charakteristischen Abkürzungen sowie bei den typischen Ellipsen im SMS-Sprachgebrauch feststellen. Am wenigsten an regionalspezifische Züge gebunden ist das Code-Switching ins Deutsche, der Dominanzsprache, die alle Domänen zumindest mitbesetzt und somit für die Rätoromaninnen und Rätoromanen zu einer Referenzsprache geworden ist, zu der jederzeit gewechselt werden kann und die auch als Brückensprache zwischen Sprecherinnen und Sprechern verschiedener Regionen zur Verfügung steht. Charakteristisch für die Situation einer Minderheitensprache sind das besondere Gewicht der Sprachkontaktphänomene infolge des Drucks der Dominanzsprache sowie die Tatsache, dass die Norm, von der man sich distanziert, von nur regionaler Tragweite ist, für die Benutzerinnen und Benutzer also bei Weitem nicht den Stellenwert erreicht, den eine "grosse" Standardsprache für ihre Benutzerinnen und Benutzer einzunehmen pflegt. Da Rätoromanisch im Allgemeinen wenig geschrieben wird, wäre es wichtig, in Erfahrung zu bringen, ob rätoromanische SMS vorwiegend von der kleinen Gruppe der häufiger rätoromanisch Schreibenden (Lehrkräfte sowie Mitarbeitende der lokalen und kantonalen Verwaltung, des Medienbereiches und der Sprach- und Kulturorganisationen) verfasst werden oder ob auch Rätoromanisch Sprechende, die sonst wenig bis gar nicht rätoromanisch schreiben, das Rätoromanische (regelmässig) in der SMS-Kommunikation verwenden. Bei Letzteren erhielte der durch die neue Kommunikationsform veranlasste schriftliche Gebrauch des Rätoromanischen eine besondere Prominenz. Der Stellenwert der SMS-Kommunikation innerhalb der allgemeinen Schreibgewohnheiten müsste im Rahmen einer neuen Datenerhebung untersucht werden, die speziell auf die Erforschung der Situation der Minderheitensprache in ihrer Koexistenz mit der Dominanzsprache ausgerichtet wäre.

Neue Datenerhebungen drängen sich auch aufgrund der aufgezeigten Nichtrepräsentativität des rätoromanischen Samples sowie angesichts des bescheidenen Umfangs des Subkorpus rätoromanischer SMS auf. Letzterer Aspekt ist wegen der Zersplitterung des Rätoromanischen in regionale, z.T. stark voneinander divergierende Varietäten doppelt nachteilhaft: Je nach Untersuchungsgegenstand wird die Datenbasis nochmals eingeengt. Dies betrifft z.B. die Ebene der Morphosyntax, wo bisherige Untersuchungen deutlich gemacht haben, dass das sprachliche Funktionieren sowie Normtendenzen regionenspezifisch zu erforschen sind. Eine Unterteilung in regionale Subkorpora drängt sich auch bei Phänomenen des Sprachkontakts auf, der in jeder Region Romanischbündens seine Ausprägung hat. Nicht 
nur das Vorhandensein eines Sprachkontaktphänomens ist relevant, sondern auch seine - je nachdem unterschiedliche - Stellung innerhalb der regionalen Varietäten.

Für die Erforschung des Rätoromanischen in der computervermittelten Kommunikation wären auch Daten $\mathrm{zu}$ anderen Kommunikationsformen $\mathrm{zu}$ sammeln, die zudem als Vergleichsbasis zu den Daten zur SMS-Kommunikation von Interesse sein können. Wir denken dabei vor allem an Daten aus Internetplattformen, die von Vereinen und Institutionen in Graubünden betrieben werden. Besondere Bedeutung für die Gewinnung rätoromanischen Materials kommt hier z.B. den elektronischen Gästebüchern zu. Erwähnt seien insbesondere Gästebücher von bündnerischen Jugendvereinen, deren Einträge oft einen informellen, situationsgebundenen Sprachgebrauch widerspiegeln und manchmal chat-ähnliche Sequenzen bilden. ${ }^{42}$ Ergiebig sind auch Gästebücher von Sportvereinen, Frauenvereinen, Fasnachtsvereinen und Websites von Institutionen wie z.B. Radio e Televisiun Rumantscha (RTR), das für die rätoromanische Schweiz zuständige Tochterunternehmen der Schweizerischen Radio- und Fernsehgesellschaft (SRG) (siehe unter www.rtr.ch). Zudem können Einträge auf Facebook, Twitter und MySpace interessante Sprachdaten zum Rätoromanischen liefern. Allerdings setzt diese Datenerhebung das Einholen der Zustimmung der Kommunikationspartner, deren Texte verwendet werden sollten, voraus, ist also wegen der eingeschränkten Zugänglichkeit der Daten mit einem besonderen Aufwand verbunden. ${ }^{43}$ Viele der genannten Internetplattformen sind auch deshalb von Interesse, weil sich hier die Stellung der Minderheitenspache Rätoromanisch im Kontext von Mehrsprachigkeit zeigt und der Umgang der rätoromanisch Schreibenden mit dieser Situation unmittelbar erfasst werden kann.

\section{Literatur}

BFS $2000=$ Personenfragebogen. Volkszählung 2000. Neuchâtel: Bundesamt für Statistik. [= http://www.bfs.admin.ch/bfs/portal/de/index/infothek/erhebungen_quellen/blank/blank /vz/fragebogen.html, Stand 19.9.2011].

Caduff, Léonard (1952): Essai sur la phonétique du parler rhétoroman de la Vallée de Tavetsch (Canton des Grisons - Suisse). Bern: Francke.

Caratsch, Reto (1983): "La Renaschentscha dals Patagons". In: ders.: Ouvras. Samedan, Stamparia Engiadinaisa: 17-118.

Coray, Renata (2009): Rätoromanische Sprachbiographien. Sprache, Identität und Ideologie in Romanischbünden. [= http://www.nfp56.ch/d_projekt.cfm?Projects.Command= download\&file=26_02_2009_02_05_57-Schlussbericht_Coray.pdf\&name $=$ Schlussbericht Coray.pdf, Stand 19.9.2011].

$\overline{D R G}=$ Dicziunari Rumantsch Grischun. Cuoira: Institut dal Dicziunari Rumantsch Grischun $1939 \mathrm{ff}$.

42 Cf. etwa die Websites der Vereine von Andiast, Rueun, Ruschein/Ladir, Samedan, Sent und Sta. Maria/Valchava (Links unter www.giuru.ch > Uniuns da giuventetgna [Stand 19.9.2011], auf den Websites der einzelnen Vereine jeweils die Rubrik cudisch [da hosps/visetas] / cudesch [da giasts] / guestbook).

43 Als weitere Quellen zur Untersuchung der computervermittelten Kommunikation seien institutionelle Websites genannt, wo das Rätoromanische für Sachinformationen vor allem in den Fällen ein Gewicht hat, in denen ein sprachgebundener Auftrag besteht - beim bereits erwähnten rätoromanischen Radio und Fernsehen, bei der Lia Rumantscha, der Dachorganisation der rätoromanischen Sprach- und Kulturvereine (www.liarumantscha.ch) - sowie in den stärker rätoromanischen Gebieten auf der lokalen Verwaltungsebene: vor allem in der Surselva, etwa in Laax, Disentis oder Tujetsch, sowie im Gebiet des Vallader, etwa in Scuol, Zernez oder in der Gemeinde Val Müstair (Links unter www.afg.gr.ch > Themen/Projekte > Informationen über Gemeinden $>$ Gemeindeliste, Stand 19.9.2011). Insgesamt marginal - auch wenn im Ausbau begriffen - ist die Präsenz des Rätoromanischen auf den Websites der Ämter des dreisprachigen Kantons Graubündens (www.gr.ch, Stand 19.9.2011), wo die rätoromanische Fassung von Eingangsseiten sowie von ausgewählten Informationen eher eine symbolische Funktion erfüllt (cf. Grünert et al. 2008: 355-358). 
Decurtins, Alexi (1958): Zur Morphologie der unregelmässigen Verben im Bündnerromanischen. Bern: Francke.

Derungs, Silvana (2009): Hey, mann, Punts goes on. Cun tge meds vegn il linguatg da giuventetgna reproduci ed imità en la gasetta "Punts"? Fribourg: Lavur da licenziat (unveröffentlicht).

Diekmann, Erwin (1982): "Italienisches Wortgut im Engadinischen, vermittelt durch sozioökonomische Wanderbewegungen". In: Winkelmann, Otto/Braisch, Maria (eds.): Festschrift für Johannes Hubschmid zum 65. Geburtstag. Bern/München, Francke: 535549.

Dürscheid, Christa (2002): "E-Mail und SMS - ein Vergleich". In: Ziegler, Arne/Dürscheid, Christa (eds.) (2002): Kommunikationsform E-Mail. Tübingen, Stauffenburg: 93-114.

Dürscheid, Christa (2003): "Syntaktische Tendenzen im heutigen Deutsch". Zeitschrift für Germanistische Linguistik 32: 327-342.

Dürscheid, Christa/Spitzmüller, Jürgen (2006): "Jugendlicher Sprachgebrauch in der Deutschschweiz: eine Zwischenbilanz". In: Dürscheid, Christa/Spitzmüller, Jürgen (eds.): Zwischentöne. Zur Sprache der Jugend in der Deutschschweiz. Zürich, Verlag Neue Zürcher Zeitung: 13-48.

Furer, Jean-Jacques (1996): Le romanche en péril? Évolution et perspective. Bern: Bundesamt für Statistik. (Recensement fédéral de la population 1990).

Furer, Jean-Jacques (2005): Die aktuelle Lage des Romanischen. Neuchâtel: Office fédérale de la statistique. (Eidgenössische Volkszählung 2000).

Ganzoni, Gian Paul (1977): Grammatica ladina. Grammatica sistematica dal rumauntsch d'Engiadin' Ota per scolars e creschieus da lingua rumauntscha e tudas-cha. Cuira: Lia Rumauntscha.

Gross, Manfred (2004): Rätoromanisch. Facts \& Figures. Chur: Lia Rumantscha.

Grünert, Matthias (2003): "Pressesprache und Standardisierung: Engadin und Surselva im 20. Jahrhundert". Ladinia 26/27: 21-40.

Grünert, Matthias (2006): "Bündnerromanische Schriftnormen. Volkssprachliche und neolateinische Ausrichtungen in Romanischbünden zwischen der Mitte des 19. Jahrhunderts und den 1930er Jahren". Vox Romanica 64: 64-93.

Grünert, Matthias et al. (2008): Das Funktionieren der Dreisprachigkeit im Kanton Graubünden. Tübingen/Basel: Francke.

Grünert, Matthias (2009): "Ein italienischer Streifzug durch die Rumantschia". Mitteilungen VBK ikg 10: 23-27.

Kaiser, Georg A. (2003): "Die Verb-Zweit-Stellung im Rätoromanischen. Ein typologischer Vergleich". Ladinia 26/27: 312-334.

Kaiser, Georg A./Hack, Franziska Maria (2010): "Sujets et sujets nuls en romanche". In: Iliescu, Maria et al.: Actes du XXV Congrès International de Linguistique et de Philologie Romanes (Innsbruck, 3-8 septembre 2007). Berlin, De Gruyter, vol. 7: 83-91.

Koch Peter/Oesterreicher Wulf (2001): "Gesprochene Sprache und geschriebene Sprache / Langage parlé et langage écrit". In: Holtus, Günter/Metzeltin, Michael/Schmitt, Christian (eds.): Lexikon der Romanistischen Linguistik. Vol. 1.2. Tübingen, Niemeyer: 584-627.

Kristol, Andres Max (1985): "Zu den italienisch-deutschen Lehnwortdubletten im Bündnerromanischen". Vox Romanica 44: 105-124.

Linder, Karl Peter (1987): Grammatische Untersuchungen zur Charakteristik des Rätoromanischen in Graubünden. Tübingen: Narr.

$L I R=$ Lexicon istoric retic, www.e-lir.ch, Stand 19.9.2011.

Liver, Ricarda (2010): Rätoromanisch. Eine Einführung in das Bündnerromanische. Tübingen: Narr.

Mani, Curo (1977): Pledari sutsilvan rumàntsch-tudestg/tudestg-rumàntsch. Cuira: Leia Rumàntscha. 
Neuland, Eva (2008): Jugendsprache. Eine Einführung. Tübingen: Francke.

Pledari grond. Cuira, Lia Rumantscha: www.pledarigrond.ch, Stand 19.9.2011.

Popovici, Victoria (2006): "Innerromanische Sprachkontakte: Romanisch und Bündnerromanisch". In: Ernst, Gerhard et al. (eds.): Romanische Sprachgeschichte. Ein internationales Handbuch zur Geschichte der romanischen Sprachen. Vol. 2. Berlin/New York, De Gruyter: 1751-1758.

Reh, Sabine (2003): Berufsbiographische Texte ostdeutscher Lehrer und Lehrerinnen als "Bekenntnisse". Interpretationen und methodologische Überlegungen zur erziehungswissenschaftlichen Biographieforschung. Heilbrunn: Klinkhardt.

Riatsch, Clà (1998): Mehrsprachigkeit und Sprachmischung in der neueren bündnerromanischen Literatur. Chur: Bündner Monatsblatt.

Rupp, Theodor (1963): Lautlehre der Mundarten von Domat, Trin und Flem, zur Bestimmung der Lautgrenzen am Flimser Wald und beim Zusammenfluss des Vorder- und Hinterrheins. Chur: Sulser.

Schlobinski, Peter/Kohl, Gaby/Ludewigt, Irmgard (1993): Jugendsprache. Fiktion und Wirklichkeit. Opladen, Westdeutscher Verlag: 93-148.

Schmid, Heinrich (1982): Richtlinien für die Gestaltung einer gesamtbündnerromanischen Schriftsprache Rumantsch Grischun. Cuira: Lia Rumantscha.

Schmid, Stephan (2010): "Symbol- und Signalphonetik. Zur linguistischen Relevanz akustischer Messungen". In: Galliker, Esther/Kleinert, Andrea (eds.): Messen in der Linguistik. Baltmannsweiler, Schneider Verlag: 71-86.

Schorta, Andrea (1946): Rätoromanische und rätolombardische Mundarten. Frauenfeld: Huber \& Co. (= Schweizer Dialekte in Text und Ton. Begleittexte zu den Sprechplatten des Phonogramm-Archivs der Universität Zürich).

Solèr, Clau (1998): "Sprachkontakt = Sprachwechsel. Deutsch und Romanisch in Graubünden". In: Werlen, Iwar (ed.): Mehrsprachigkeit im Alpenraum. Aarau etc., Sauerländer: 149-163.

Solèr, Clau (1999a): "Sprachwandel als Zeichen der Vitalität - oder als Vorzeichen des Sprachwechsel". In: Kattenbusch, Dieter (ed.): Studis romontschs. Beiträge des Rätoromanischen Kolloquiums (Gießen/Rauischholzhausen, 21.-24. März 1996). Wilhelmsfeld, Egert: 95-108.

Solèr, Clau (1999b): "Überwindung der Sprachgrenzen - zurück zur Realität". In: Reiter, Norbert (ed.): Eurolinguistik. Ein Schritt in die Zukunft. Wiesbaden, Harrassowitz: 289302.

Solèr, Clau (2003): "Gesteuerte und spontane Spracherneuerung. Das Bündnerromanische im Alltag". Ladinia 26/27: 103-20.

Solèr, Clau (2004): "Le Rhéto-Romanche en Suisse. Bilinguisme et Diglossie: Problèmes et propositions". Education et Sociétés Plurilingues 16: 15-26.

Solèr, Clau (2010): "Bilinguisme déséquilibré - le cas du Rhétoroman en Suisse". In: Guzman, Josep R./Verdegal, Joan (eds.): Minorised languages in Europe: State and Survival. Compostela, Group of Universities: 408-424.

Spescha, Arnold (1989): Grammatica sursilvana. Cuera: Casa editura per mieds d'instrucziun.

Strebel, Barbara (2005): Il riflessivo in soprasilvano. Indagine di morfosintassi sincronica e diacronica. Zürich: Lizentiatsarbeit (unveröffentlicht).

Widmer, Ambros (1963): "Beiträge zur Mundart von Medels". Vox Romanica 22: 177-191.

Wunderli, Peter (1993): "Requiem für eine heilige Kuh. Das "Neutrum" im Surselvischen". Annalas da la Societad Retorumantscha 106: 134-163. 